\title{
The Lublin Protocol of the Uterine Arteries Embolization in the Treatment of Symptomatic Uterine Fibroids
}

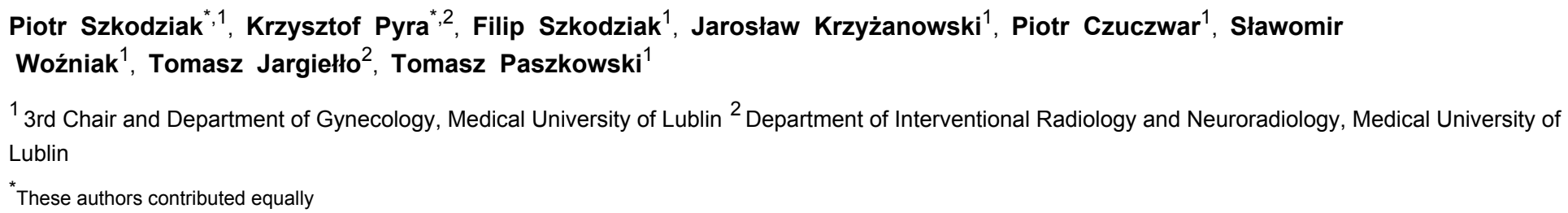

\section{Corresponding Author}

Piotr Szkodziak

piotr.szkodziak@gmail.com

\section{Citation}

Szkodziak, P., Pyra, K., Szkodziak, F., Krzyżanowski, J., Czuczwar, $\mathrm{P}$. Woźniak, S., Jargiełło, T.,

Paszkowski, T. The Lublin Protocol of the Uterine Arteries Embolization in the Treatment of Symptomatic Uterine Fibroids. J. Vis. Exp. (163), e61530, doi:10.3791/61530 (2020).

\section{Date Published}

September 15, 2020

DOI

$10.3791 / 61530$

URL

jove.com/video/61530

\section{Abstract}

Uterine fibroids are benign tumors originating from smooth muscle tissue, constituting uterine muscle stroma. Uterine fibroids are the most common benign tumors found in women. In $20 \%-50 \%$ of women, fibroids are asymptomatic and do not require any treatment. The main symptoms of uterine fibroids are profuse menstrual bleeding, abnormal uterine bleeding, and pressure symptoms. Pressure symptoms can cause pelvic pain syndrome, urination disorders, and constipation.

The treatment methods that are currently used include surgical treatment, pharmacological therapy, and minimally invasive procedures. The most commonly applied minimally invasive method is the embolization of uterine arteries. This procedure is currently a widely accepted method of treatment for symptomatic uterine fibroids and has been recognized as such by the National Institute for Health and Clinical Excellence in the guidelines for heavy menstrual bleeding.

This is a complicated procedure and requires close cooperation between gynecologists and interventional radiologists. We present a protocol applicable to uterine artery embolization in the treatment of symptomatic uterine fibroids. The protocol is divided into five section. The first two section are intended for gynecologists and interventional radiologists, explaining how to qualify and prepare a patient for embolization in a step-by-step manner. Section three, which is directed at interventional radiologists, explains how embolization should be done. Section four is directed at gynecologists or hospital ward doctors who look after the patients after embolization. This section of the protocol offers a method for treating postembolization pain using the Patient Controlled Analgesia (PCA) pump. Section five completes the procedure with an assessment of the effects and late complications of uterine artery embolization. 
All five section create a uniform protocol directed at clinicians, experts, and researchers new to the field.

\section{Introduction}

Uterine fibroids are benign tumors originating from smooth muscle tissue, constituting uterine muscle stroma. They are monoclonal tumors, consisting of a large amount of extracellular substance containing collagen, fibronectin, and proteoglycans. The fibroids are surrounded by a thin pseudocapsule made of compressed muscle fibers, collagen fibers, neurofibers, and blood vessels ${ }^{1,2}$. The pathophysiology of myomas is not entirely understood but seems to rely mainly on monoclonal proliferation caused by selective and tissue-specific epigenetic changes ${ }^{3}$. No single gene was found to cause uterine fibroids. However, the presence of rare uterine fibroid syndromes, such as multiple cutaneous and uterine leiomyomatosis, has been attributed to a gene that codes for fumarate hydratase, a mitochondrial enzyme involved in the Krebs cycle ${ }^{4}$. The presence of chromosome 7 deletions and translocations in chromosomes 7,12 , and 14 , which occur in $50 \%$ of fibroids, seems to be secondary rather than primary $5,6,7$.

The regulators of the growth of uterine fibroids are steroid hormones produced by ovaries (estrogens and progesterone), growth factors, angiogenesis, and apoptosis. Risk factors for the development of uterine fibroids have also been identified, including age, early menarche, AfricanAmerican race, heredity, nulliparity, obesity, polycystic ovary syndrome, diabetes, hypertension, vitamin D deficiency, use of soybean milk, alcohol, and caffeine consumption ${ }^{8}$.

Uterine fibroids are the most common benign tumors of reproductive organs in women. These tumors were first described in 1793 by Matthew Baillie at St George's Hospital in London. Available epidemiological data do not accurately specify the incidence of uterine fibroids, as their large proportion remain undiagnosed. It is estimated that uterine fibroids occur in $5.4 \%$ to $77 \%$ of all patients. Their prevalence is higher in the United States than in Europe, the probable cause being racial differences ${ }^{8}$.

Among women in the childbearing age, approximately $30 \%$ of myomas may give clinical symptoms in the form of abnormal uterine bleeding, resulting in inadequate blood supply in patients $^{9}$. In most cases, patients have more than one myoma, which are spherical lesions located in the uterus. Their dimensions and location may vary. In $90 \%$ of the cases, they are located in the body of the uterus. Their diameter can be from several millimeters to $20 \mathrm{~cm}^{10}$.

The FIGO (Fédération Internationale de Gynécologie et d'Obstétrique) classification divides them into groups from 0-8 depending on the proximity of the endometrium (lower the number, closer will be the endometrium) (Figure 1) ${ }^{11}$. In about $50 \%-75 \%$ of the cases, fibroids are asymptomatic. The most common symptoms of uterine fibroids are profuse menstrual bleeding, abnormal uterine bleeding, and pressure symptoms. Myomas are associated with about $10 \%$ of the cases of infertility, and in $1 \%-3 \%$ they are the only cause $^{12}$. Asymptomatic uterine fibroids usually undergo only a regular medical control, whereas symptomatic fibroids are an indication for treatment ${ }^{13}$.

The treatment methods for uterine fibroids that are currently used include surgical treatment, pharmacological therapy, 
and minimally invasive procedures ${ }^{13}, 14,15,16,17,18$. Surgical treatment includes myomectomy (abdominal and hysteroscopic) and hysterectomy. Both myomectomy and hysterectomy have a positive impact on the quality of life ${ }^{19}$. Hysterectomy is associated with irreversible loss of fertility; thus, many women seek other treatment options ${ }^{20}$.

Abdominal myomectomy allows for the preservation of fertility. Depending on the size and number of fibroids, as well as the experience of the surgeon, this procedure can be performed via laparotomy or laparoscopy. Although hemorrhages are less common than in hysterectomy, overall morbidity is similar. Hysteroscopic myomectomy is a safer, less invasive method than abdominal myomectomy and allows the treatment of submucous fibroids (FIGO 0 ). Subsequent hysteroscopic procedures may be necessary to completely remove larger type-2 fibroids ${ }^{21}$.

Levonorgestrel releasing intrauterine devices are an effective treatment for heavy menstrual bleeding, but they do not reduce the size of the fibroids. Their use is limited in patients with the deformed uterine cavity. GnRH analogues are mainly used as a pre-operative agent to reduce the size of fibroids and perioperative blood loss. They also reduce the percentage of vertical incisions during hysterectomy and myomectomy while increasing the possibility of a vaginal procedure $^{20}$.

In the short term, selective progesterone receptor modulators reduce the myoma volume and induce amenorrhea. However, the long-term efficacy and safety require further research. Along with aromatase inhibitors, there may be other options for pre-operative treatment of anemia and reduction of the myoma volume ${ }^{22}$. Some studies suggest that vitamin
D may slow down or prevent the growth of fibroids and the onset of symptoms ${ }^{23}$.

New methods using 2-methoxyestradiol combined with nanoparticles are also under development ${ }^{24}$. Minimally invasive methods used in the treatment of fibroids include uterine artery embolization (UAE), magnetic resonanceguided focused ultrasound surgery (MRgFUS), laparoscopic uterine artery occlusion (LUAO), and radiofrequency myolysis $^{14,25}$. Ultrasound-guided High-Intensity Focused Ultrasound (US-HIFU) is a new, still experimental, minimally invasive method 26,27 .

Methods of therapeutic vascular occlusion and blocking the blood supply to the uterus were mentioned over 120 years ago. In 1894, Kelly presented the ligation of internal iliac arteries during an oncological hysterectomy to control intractable pelvic bleeding, which at that time was a common complication after the surgical procedure ${ }^{28}$. Then, Sack (1973) described the effective use of the same technique in the treatment of massive postpartum hemorrhage after forceps delivery. In both cases, hemostasis was achieved without hysterectomy ${ }^{29}$. In 1979, Heaston et al. and Brown et al., independently described embolization of the pelvic arteries using absorbable gelatin sponges to control postpartum hemorrhage 30,31 .

UAE was first used as a treatment method for symptomatic uterine fibroids in 1991 in France ${ }^{32}$. It was initially used to reduce blood loss after myomectomy. In 1995, Ravina et al. proposed this procedure as the primary treatment method for symptomatic uterine fibroids ${ }^{33}$. In the United States, uterine artery embolization was successfully performed in $1997^{34}$.

The growing interest in uterine preservation in women with symptomatic fibroids put UAE at the forefront of minimally 
invasive fibroids treatment ${ }^{14,18,35,36,37}$. In 2000, the Joint Working Party of the Royal College of Obstetricians and Gynecologists and The Royal College of Radiologists was created to issue guidelines on UAE. At that time, UAE was considered to be an experimental method (less than 7,000 procedures performed worldwide). Since the guidelines were published, more than 100,000 UAE procedures have been performed worldwide. Also, five randomized controlled trials have been carried out, comparing the UAE with other surgical procedures. Research results indicate that UAE is highly effective in the short-to-medium term (up to a few years) with a low risk of medium (e.g., uterine infection) and serious (life-threatening) complications ${ }^{38,39}$. In randomized studies, shorter hospitalization times, faster recovery, and return to daily activities speak in favor of UAE. Surgical procedures proved to be cheaper and less frequently required re-intervention while maintaining statistical significance ${ }^{32}$. This procedure is currently a widely accepted method of treatment for symptomatic uterine fibroids and has been recognized as such by the National Institute for Health and Clinical Excellence (NICE) in the guidelines for heavy menstrual bleeding ${ }^{40}$.

Currently, there are 11 recommendations regarding the use of UAE in the treatment of symptomatic uterine fibroids, created by scientific societies from Europe, North America, and Australia. In most cases, the recommendations are consistent while the divergence concerns two discrepancies. The first is whether pedunculated submucosal (FIGO 0 ) and subserosal (FIGO 7) myoma are contraindications for UAE. The second is whether women declaring future pregnancy should be qualified for this procedure ${ }^{41}$. The American College of Obstetricians and Gynecologists (ACOG) (2008) presented an interesting guideline for the treatment of uterine fibroids. Based on consistent scientific evidence (Level A), the ACOG defined UAE as an effective and safe method for appropriately qualified women who wish to preserve the uterus with typical indications for the treatment of fibroids. At the same time, the ACOG recommendations emphasized the need for close cooperation between gynecologists and interventional radiologists. In the published guidelines, the ACOG recognized the desire to preserve fertility as the only contraindication (relative) ${ }^{42}$.

One of the latest recommendations was issued in 2013 by the Royal College of Obstetricians and Gynecologists (RCOG) and in 2015 by the Society of Obstetricians and Gynecologists of Canada (SOGC) ${ }^{41}$. In the remaining part of this article, the authors will use the above recommendations. According to the guidelines of RCOG and SOGC, any patient with symptomatic myomas can be a candidate for embolization, provided there are no contraindications and the benefits of the procedure (resolution of symptoms) outweigh the risk of complications. It should be noted that the embolization of uterine fibroids as a minimally invasive procedure carries a negligible number of serious complications. Hence, the benefits in most cases outweigh the risk of complications ${ }^{14,32,43}$.

An appropriate patient qualification is of crucial importance for high clinical effectiveness and prevention of complications after UAE. The main indication for UAE is symptomatic uterine fibroids, resulting in heavy menstrual bleeding, dysmenorrhea, pain, dyspareunia, and other adverse effects on the urinary or gastrointestinal tract. It is necessary to differentiate fibroids from adenomyosis or fibroids co-existing with adenomyosis because, in such a situation, UAE is less effective and requires modification of the procedure technique $14,32,43,44,45,46,47$. Specific indications for performing the UAE procedure in women with 
symptomatic uterine fibroids include refusal of surgery, no consent for blood transfusion, and previously failed uterine fibroids surgery.

In the above indications, UAE should be treated as an alternative to surgical treatment. However, patients should be advised that in a small number of cases, complications after the procedure may result in the need for a surgical intervention $14,32,43$. According to the RCOG guidelines, the use of UAE in a situation in which myoma is a probable cause of infertility requires special care and proper assessment of a gynecologist specializing in the treatment of infertility and assisted reproduction. Infertility due to the presence of fibroids is not absolute, and many women will become pregnant without any intervention. Therefore, it is reasonable to exclude other possible causes of infertility, including assessment of the male partner ${ }^{14,32,43}$.

Thus, according to the recommendations of RCOG and SOGC, the candidates should be women with symptomatic uterine fibroids in whom pathologies in the pelvis with fibroidlike clinical symptoms have been excluded ${ }^{41,43}$.

Absolute contraindications to this procedure include current or recent genital infection, diagnostic doubts due to clinical factors or inadequate imaging, asymptomatic fibroids, viable pregnancy, and contraindications for the use of radiological contrast agents.

Relative contraindications include pedunculated submucosal (FIGO 0) and subserosal (FIGO 7) fibroids, which theoretically may detach from the endometrium due to peduncle necrosis, in rare cases resulting in sepsis. In these cases, UAE should only be considered if hysteroscopic or laparoscopic removal of pedunculated myoma before the procedure is planned.
Despite the fact that current literature suggests that the size of the myoma is not a contraindication by itself, experience shows that extreme caution is required when qualifying patients with large myomas (especially associated with compression symptoms) for UAE, as the reduction in volume may be insufficient to relieve symptoms and meet patient expectations $^{14,32,43,48}$.

There are many reports of successful pregnancy after UAE, but existing evidence does not fully support its use as an alternative to pharmacological or surgical treatment (myomectomy) in young women ${ }^{49}$. Therefore, this procedure should be used with great caution in women who declare a desire to become pregnant (as there is a lower pregnancy rate, a higher miscarriage rate, uterine rupture, placenta accreta, and adverse pregnancy outcomes after UAE than after myomectomy) $32,50,51,52,53,54,55$. As gynecologists, we do not recommend UAE for women seeking pregnancy. For us, it is a relative contraindication, provided that there are additional indications for UAE, such as refusal for surgery or blood transfusions, where the lack of treatment may be lifethreatening.

According to RCOG guidelines, the desire to preserve or improve fertility in young women with symptomatic uterine fibroids is a relative contraindication to $U A E^{32}$. In contrast, SOGC guidelines recommend that in similar cases UAE should not be proposed as a treatment option for fibroids, because safety and effectiveness in such women have not been established ${ }^{14,43}$. A similar point of view is represented by other scientific societies, including American Society for Reproductive Medicine (ASRM), American College of Obstetricians and Gynecologists (ACOG), American College of Radiology (ACR), Royal Australian and New Zealand College of Obstetricians and 
Gynecologists (RANZCOG) and others, citing improved fertility outcomes after myomectomy $41,43,56$. UAE has been recommended only by NICE for women seeking to maintain or improve fertility, especially with features unfavorable to myomectomy (multiple fibroids) ${ }^{57}$.

Generally, the procedures of UAE could be performed at any stage of the menstrual cycle $^{32}$. However, assuming that there is no ideal method to exclude pregnancy at the stage of fertilization or implantation, in order to exclude early pregnancy, in our center, it is performed until the 10th cycle day. In most cases, patients are admitted to the gynecology ward on the day of the procedure. Admitting a patient to the vascular surgery ward is permissible if appropriate examinations and gynecological consultation are provided. A gynecologist and an interventional radiologist perform qualification for embolization of fibroids. Gynecological qualification includes medical history, examination, an ultrasound assessment of the reproductive organ and the myoma type. In addition, to exclude any malignancy within the uterus, a cervical (PAP) smear and endometrial biopsy are necessary. In cases where the ultrasound scan of the ovaries is questionable, ROMA test (Risk of Ovarian Malignancy Algorithm) is necessary.

A separate issue requiring discussion is uterine sarcoma, in particular leiomyosarcoma (LMS), which accounts for $70 \%$ of these uterine tumors. The prevalence of LMS in patients operated on for myoma is low and is estimated at $0.13 \%-0.29 \% 58,59$. The increase in the incidence of LMS is observed in women over 40 years of age. LMS is difficult to diagnose before treatment as it may resemble benign fibroids ${ }^{60}$. Most LMSs are unrelated to pre-existing fibroids, and there is no evidence of an association of LMS with uterine fibroids ${ }^{61}$. Both uterine fibroids and LMS tend to grow rapidly.
Thus, the size or growth rate is not a risk factor for a malignant uterine tumour 60 .

Currently, there are no reliable laboratory or imaging tests that would allow us to clearly identify leiomyosarcoma and differentiate it from leiomyoma ${ }^{60,62}$. The sensitivity of endometrial biopsy in the diagnosis of leiomyosarcoma is $86 \%$. Thus, a negative biopsy result does not exclude the existence of a malignant uterine tumor. Contrast-enhanced MRI is currently the optimal diagnostic method for uterine tumors. The sensitivity of this test in the diagnosis of LMS is $94 \% 60$.

As already mentioned, the above tests do not exclude $100 \%$ of malignant uterine tumors. Therefore, there is a slight risk of prolonging the diagnosis of LMS after treatment, without the possibility of histopathological verification of the uterine tumor. The patient should be informed about this during the qualification for UAE.

Performing a complete blood count (CBC) and coagulation tests (INR, APTT), renal panel (creatinine, urea), thyroidstimulating hormone (TSH), the concentration of antiMullerian hormone (AMH) (recommended) or the folliclestimulating hormone $(\mathrm{FSH})$ at follicular phase, C-reactive protein (CRP), general urine test, and vaginal smear (aerobic vaginal culture) make it possible to assess and avoid potential post-embolization complications (infections, iatrogenic ovarian damage, intensification of previous renal impairment after Gadolinium-based contrast agents, thyrotoxicosis in cases of hyperthyroidism after an iodinebased contrast agent ${ }^{63,64}$. Please note that $\mathrm{FSH}$ testing is not recommended under the age of 40 , as FSH is not a sensitive indicator of changes in ovarian reserve in young women 50,65 . 
An interventional radiologist qualifies patients for the procedure based on medical history and magnetic resonance imaging (MRI). While gathering medical history, the benefits and possible complications, as well as the procedure itself, should be discussed with the patient. Patient's expectations regarding UAE should also be discussed. MRI aims to exclude other pathologies of the reproductive organ and adjacent structures as well as assessing the morphology and location of fibroids and anatomy for the technical feasibility of the procedure $35,36,37,57$.

UAE aims to completely block the vasculature of all myomas while maintaining the blood supply to the uterus, ovaries, and surrounding tissues within the pelvis. The technical aspects of UAE are still evolving to some extent.

The embolization of uterine arteries is performed by interventional radiologists with appropriate competence in the field of intravascular embolization. The procedure is carried out under fluoroscopy guidance. It involves the percutaneous insertion of a vascular catheter from the puncture in the inguinal region into the femoral artery, the aorta, the internal iliac artery, up to the uterine artery. After placing the catheter deep in the uterine artery and achieving a stable position, the embolization agent mixed with contrast is injected under fluoroscopic control in such a way as to avoid reflux and "nontarget" embolization. The vascular bed of myomas is closed using particles of 500-900 $\mu \mathrm{m}$ depending on the type of embolization material-usual sizes are $700 \mu \mathrm{m}$. Embolization is continued until stasis blood flow is achieved. At the end of the procedure, the catheter is removed, and the vascular access site is secured with manual pressure and dressing or mechanical closer. The procedure lasts for approximately 0.5-1.0 $\mathrm{h}$. The mean ionizing radiation dose absorbed by the ovary during UAE ranges from 0.04-0.22 (Gy: gray) and mean estimated effective dose ranges from 22-34 (mSv: millisievert). Mean fluoroscopic time is about $22 \min ^{66,67}$.

The vasculature of most fibroids comes from the uterine arteries. Only about $5 \%-10 \%$ of fibroids are additionally supplied by ovarian arteries. Arterial uterine anastomoses occur in about $10 \%$ of cases, while utero-ovarian in $10 \%-30 \%$. Cutting off the blood supply to the embolized tissues causes ischemic necrosis, followed by hyaline degeneration or coagulative necrosis. This process takes several months 68,69 .

The effectiveness of UAE depends on the resolution of symptoms or the degree of their reduction. For the treatment of excessive menstrual bleeding, pelvic pain, and pressure symptoms, the clinical effectiveness index for UAE is $81 \%-$ $96 \%, 70 \%-100 \%$, and $46 \%-100 \%$, respectively. Within 36 months after the procedure, the observed reduction in the volume of fibroids was $25 \%-60 \% 33,70,71,72$. The mean diameter reduction of the myoma was $2.2 \mathrm{~cm}^{57}$.

The reduction in the volume of fibroids does not always correlate with the resolution or reduction of clinical symptoms. In long-term follow-up after UAE, more than $70 \%$ of patients reported resolution or significant reduction of clinical symptoms within 5 years after the procedure, while $16 \%-23 \%$ of them required re-intervention 73,74 .

In assessing the early UAE effect, authors of this publication propose the use of three-dimensional (3D) ultrasound, using Virtual Organ Computer-aided AnaLysis (VOCAL), performing measurements of vascular indices: vascularization index $(\mathrm{VI})$, flow index $(\mathrm{FI})$, and vascularization flow index $(\mathrm{VFI})^{75}$.

Post-embolization pain is an early (lasting about $24 \mathrm{~h}$ ) expected after-effect of successful UAE (not to be confused 
with a complication) and should be actively treated. This clinical symptom is caused by the release of tissue breakdown products of ischemic myoma. The treatment includes adequate pain control, hydration, and possible antibiotic therapy $^{32,43}$. Epidural anesthesia (EA) maintained for $24 \mathrm{~h}$ after the procedure significantly reduces pain to a completely acceptable level, but at a higher cost and increased risk of complications compared to Patient-Controlled Analgesia $(\mathrm{PCA})^{76}$.

Other approaches are also available in post-embolization pain management reviews. It is worth mentioning the use of mixtures of pain medications with polyvinyl alcohol molecules and electroacupuncture anesthesia during UAE. Both methods were intended to limit the number of UAE procedures performed in a hospital setting ${ }^{77,78}$. We do not use these methods in our and cooperating centers.

The early complications of UAE are usually local complications associated with the angiography procedure. This group of adverse events is rare (they occur in less than $1 \%$ of cases) and relates mainly to groin hematoma, arterial thrombosis, arterial dissection and pseudo-aneurysm, allergic reactions to contrast agents, a spasm in the uterine artery caused by manipulation of the catheter in the vessel during the procedure (treated as a temporary event after a few minutes pass and the procedure can be continued, if the spasm persists, Verapamil $(2.5-5 \mathrm{mg})$ or Nitrate (100-150 $\mu \mathrm{g})$ intraarterially could be given) and "non-target" embolization $32,37,38$.

There have also been several reports of "non-target" embolization of other pelvic organs and their subsequent ischemia. This complication can occur as a result of poor execution of the procedure, as well as due to the presence of anastomoses and anatomical variants of the pelvic vasculature. A special case of non-target embolization is ovarian damage that results from anastomoses between the vessels of the uterus and the ovaries in some patients ${ }^{79,80}$.

The consequence of myoma necrosis is a post-embolization syndrome occurring within 30 days of the procedure in approximately $10 \%-15 \%$ of patients. The symptoms of this syndrome, which may occur together or individually, include nausea, vomiting, malaise, low-grade fever, lower abdominal pain, and elevated leukocyte levels. This is usually a self-limiting syndrome that usually disappears within 10 14 days. Analgesics and anti-inflammatory drugs are used to treat this complication ${ }^{32}$. It is important to differentiate the symptoms of a post-embolization syndrome with more serious complications such as sepsis. This is especially true in cases where the mentioned symptoms last longer than two weeks $^{32,37,38}$.

Infection is potentially the most serious complication after UAE, and it occurs in about $0.5 \%$ of cases ${ }^{32,38}$. In a case of persistent, high fever $\left(38.5^{\circ} \mathrm{C}\right.$ and above) for $24-48$ hours and hard and painful abdomen, a sepsis should be suspected. In this case, treatment may not only require the use of antibiotic therapy, but also the need to remove the uterus. In the latter case, in less than $1 \%$ of cases, it may pose a threat to the patient's life. Sepsis is more common when UAE is performed on a large uterus (over $20 \mathrm{~cm}$ or when the diameter of a single myoma is larger than $9 \mathrm{~cm}$, and also in the case of coexistence of large submucous fibroids) ${ }^{37,38}$.

A late complication after UAE (more than 30 days after the procedure), occurring after embolization of submucous fibroids, is the excretion of demarked, necrotic fragments of fibroids through the cervical canal. It happens in about $10 \%$ of cases $32,81,82$. Approximately $16 \%$ of women, after UAE, may have abundant vaginal discharge for several weeks up 
to many months as a result of the excretion of necrotic fibroids from the uterus ${ }^{83}$. After the UAE procedure, a significant shortening of menstruation and a decrease in its abundance is observed, which is considered a beneficial effect of this procedure. However, complete amenorrhea is treated as the effect of post-embolization ovarian failure ${ }^{50,84}$.

Amenorrhea after UAE is usually transient and limited to a few cycles. Permanent amenorrhea occurs in about $15 \%$ of women over 40 years of age and in about $1 \%$ of women under this age, causing symptoms of premature menopause. In our own research, a decrease in fertility in young women (3340 years) as a result of the reduction of the ovarian reserve was found ${ }^{65}$. It is estimated that about $85 \%$ of women who report amenorrhea after UAE are over 45 years old ${ }^{85}$. It has been argued that the reason for the increase in the incidence of amenorrhea in older women is due to the reduced ovarian reserve and greater sensitivity of ovarian tissue to ischemia caused by "non-target" embolization ${ }^{86}$.

The UAE procedure also affects the sexual function of women undergoing the procedure. Improvement of sexual function after UAE was reported by $26 \%$ of women, deterioration was found in $10 \%$, and unchanged in the remaining $64 \%$ of women. A possible cause of sexual dysfunction is abnormal vasculature of the clitoris, cervix, and corpus uteri as a result of the UAE procedure ${ }^{74}$.

\section{Protocol}

The authors declare that the protocol follows the guidelines of the local Ethical Committee of the Medical University of Lublin.

\section{Qualification for the UAE procedure}

NOTE: This is addressed to gynecologists and interventional radiologists.

1. Inform the patient about the details of UAE procedure.

1. Discuss indications and contraindications for the UAE procedure.

2. Discuss with the patient the assumptions of UAE and alternative methods (described in the Introduction) in the treatment of uterine fibroids.

3. WARNING! Inform the patient that the UAE is not a radical procedure (the myoma will not be removed).

4. WARNING! Inform the patient that she will not receive a histopathology report of uterine fibroids after UAE.

5. WARNING! Inform the patient that 1-3 cases out of 1,000 uterine tumors can be malignant (LMS). Discuss with the patient that the endometrial biopsy and MRI performed during UAE qualification is highly sensitive in the diagnosis of leiomyosarcoma, but not $100 \%$. Inform the patient that performing UAE for leiomyosarcoma may delay diagnosis and proper treatment. Note that fact in the medical record.

6. If the patient knowingly wants UAE performed, continue this Protocol.

7. Inform the patient that the qualification takes place in two stages. Qualification by a gynecologist includes analysis of indications and contraindications, while an interventional radiologist assesses the technical capabilities of UAE.

8. Plan or perform a gynecological consultation and plan a consultation with the interventional radiologist before UAE. 
9. If possible, perform as many of the qualification procedures as possible at the outpatient clinic.

2. Ensure that the patient has indications for UAE: symptomatic uterine fibroids (menometrorrhagia, metrorrhagia, dysmenorrhea, dyspareunia, chronic pelvic pain); the refusal of surgery treatment; no consent for blood transfusion; previously failed uterine fibroids surgery. Note them in the medical history.

1. Ask the patient about the symptoms of fibroid(s).

2. Discuss the effectiveness of UAE and the expectations of the patient (in the Representative Results).

3. Ask whether the patient was treated for uterine fibroids previously. Ensure which method was used.

4. If the patient refuses surgical treatment or blood transfusion, take a written statement.

3. Ensure that the patient who qualified for the UAE procedure does not have absolute contraindications: asymptomatic fibroids; a viable pregnancy; current or recent genital infection; diagnostic doubts due to clinical factors or inadequate imaging; contraindications for the use of radiological contrast agents; features of leiomyosarcoma on MRI; uterine or ovarian malignant tumor unless it is performed for palliation or as an adjunct to surgery ${ }^{87}$. Note them in the medical history.

1. If the patient demands UAE despite asymptomatic fibroids, explain that such cases do not qualify for the procedure. Explain to the patient that asymptomatic fibroids only require regular gynecological monitoring.

4. If there are relative contraindications to UAE (large fibroids and reproductive plans), discuss them with the patient and make a note of that fact in the medical record.
1. Focus on the possible complications related to relative contraindication, described in the introduction, ensure the patient understands and agrees to the procedure.

5. Discuss the after-effect and complications of uterine artery embolization with the patient (see introduction) and note that fact in the medical record.

1. When talking to the patient, pay special attention to the post-embolization pain.

2. Discuss post-embolization pain treatments.

6. Perform a gynecological qualification.

1. Collect medical history and perform a gynecological examination.

2. Perform an ultrasound assessment of the reproductive organs to evaluate the size and type of fibroids (FIGO classification) ${ }^{88}$.

1. Use a vaginal probe to estimate the size and location of the myoma (Figure 2). The uterine fibroids treated with UAE should be $2-5$ (ideally, 3-4) according to FIGO classification, and the diameter should not exceed $10 \mathrm{~cm}$.

2. Include the protocol of the pelvis ultrasound scan in the medical record.

3. Ensure that the ultrasound scan result gives indications for qualification for the UAE procedure.

3. Exclude malignant processes within the pelvis.

1. Ensure that the patient has had a current (preferably performed within 6 months) results of cervical (PAP) smear and endometrial biopsy. 
2. During a pelvic ultrasound, use the vaginal probe to evaluate the ovaries and confirm that their structure is correct. If in doubt, check the previous ultrasound scan or perform the ROMA test.

3. Order an MRI to evaluate the uterine tumor for LMS features and UAE technical capabilities.

4. Note the results of the cervical (PAP) smear, the endometrial biopsy, the ultrasound scan (possibly the ROMA test), and the MRI in the medical record.

5. If the results are incorrect, disqualify the patient from the procedure.

4. Inform the patients about the results of the gynecological qualification.

1. Note that fact in the medical record. Refer the patient to the hospital.

2. Make an appointment for the UAE procedure and remember that the patient should be before the 10th day of the cycle.

3. Ask the patient if she refuses surgery. In case of refusal note that fact in the informed consent of the patient or the medical record.

4. Patients with symptomatic fibroids who refuse surgery qualify for UAE.

5. Perform the following tests at least 7 days before UAE to assess and avoid potential complications: microbiological test of vaginal smear (aerobic vaginal culture); urinalysis; selected blood tests: CBC, Coagulation tests (INR, APPT), Renal panel (creatine, urea), TSH, CRP.

1. Describe the results in the medical record.
2. Inform the patient that incorrect results disqualify her for the procedure.

6. Check the patient's ovarian reserve by testing $\mathrm{AMH}$ (recommended) or $\mathrm{FSH}$ (not recommended under 40 years) serum levels at the follicular phase. Discuss with the patient that the above test will be helpful to assess ovarian damage as a result of non-target embolization. Note that fact in the medical record.

7. Patients who are qualified for UAE should not declare the will to become pregnant due to the increased risk of pregnancy complications $^{14,32,38,50,51,52,89,90,91 .}$

1. Inform the patient about the increased risk of pregnancy complications after UAE.

2. Note the fact that the patient does not declare the will to become pregnant in the informed consent of the patient or the medical record.

3. If the patient still does not exclude pregnancy in the future, but requires $U A E$, take a written declaration that she has been informed about possible pregnancy complications after UAE.

7. Perform radiological qualification.

1. Ensure that pelvic MRI has been performed. MRI examination allows to exclude other pathologies of the reproductive organ and adjacent structures (step 1.6.3.3. of the Qualification for UAE procedure), assess the morphology and location of fibroids, and anatomy for the technical feasibility of embolization (Figure 3, Figure 4). If not, arrange the MRI examination and consultation with an interventional radiologist. 
2. Analyze the imaging examinations and assess the type and size of fibroids.

1. If submucosal (FIGO $0-1$ ) fibroids are found, inform the patient about the risk of sloughing into the uterine cavity. Complete excretion may cause sepsis, while excretion in fragments is usually asymptomatic $^{50}$.

2. If subserosal narrow-stalked, pedunculated (FIGO 7) fibroids are found, inform the patient about the risk of post-embolization detachment of the fibroid into the abdominal cavity. It can lead to acute inflammation, and laparoscopic reoperation may be needed ${ }^{92}$.

3. If large fibroids are found, inform the patient that the risk of complications remains unchanged, but the outcome of the procedure is better for small fibroids $^{93}$.

4. Note that fact in the informed consent of the patient or medical record.

\section{Preparing for the UAE procedure}

NOTE: This is addressed to gynecologists or vascular surgeons and interventional radiologists.

1. Admit the patient to the hospital (gynecological or vascular surgery department).

1. Ensure that she is before the 10th day of the cycle at admission.

2. Perform or order a gynecological examination and an ultrasound scan of the pelvis.

1. Ensure that the diagnosis and qualification for the UAE procedure are correct.
2. Ensure that no new contraindications have appeared since the qualification for UAE. If so, suggest other treatment options and change the date of the procedure.

3. Perform the 3D transvaginal ultrasound scan (TVUS) and calculate the volume and vascularization indexes of the fibroid ( $\mathrm{VI}, \mathrm{FI}$, and $\mathrm{VFI}$ ) using the VOCAL software exam (which is used to assess the effectiveness of the UAE procedure ${ }^{72,75}$ ) (described in the introduction) (Figure 5 and Figure 6).

4. Note that fact in the medical record.

3. Ensure that the patient qualified for $U A E$ has tests performed during qualification for UAE (described in steps 1.6.2, 1.6.3, 1.6.5 and 1.6.6 of Qualification for UAE).

1. If the patient has been pre-qualified by a gynecologist and does not have blood tests, a urine test, and an $\mathrm{MRI}$, order the tests and arrange a consultation with an interventional radiologist (step 1.7 of Qualification for UAE).

2. Rate or order AMH (recommended) or FSH (not recommended under 40 years) serum level before UAE to enable the assessment of ovarian damage as a result of "non-target" embolization.

3. Inform the patient that the test results will be available in about a day and the UAE procedure will be possible only after receiving these results.

4. Inform the patient that incorrect results disqualify her for the procedure.

5. WARNING! In the case of missing or incorrect results of PAP smear, endometrial biopsy; the microbiological test of vaginal smear, disqualify the 
patient from UAE (too long time to obtain these results). Re-qualify after obtaining the correct results.

6. Note that fact in the medical record.

4. Obtain an informed consent to perform UAE.

1. Provide the patient with all information about UAE.

2. Discuss the possible complications of UAE (described in the introduction).

3. Leave the patient time to think and ask questions; allow the patient to give an informed consent to perform the UAE procedure.

5. Inform the patient that she has the right to change her decision until UAE begins.

1. If the patient changes her decision regarding $U A E$, then suggest other treatment options for uterine fibroids (described in the introduction).

2. Note that fact in the informed consent of the patient or medical record.

6. Immediately before the UAE procedure, check and ensure that the tests described in sections 1.6.2, 1.6.3, 1.6.5 and 1.6.6 of Qualification for UAE as well as 3D TVUS, MRI, and consultation with an interventional radiologist have been performed, and the results are correct.

1. Disqualify a patient from UAE if the tests are missing or results are incorrect.

2. Note that fact in the medical record.

7. Inform the patient and take care of the prohibition on taking food and liquids from morning until the procedure. The exception is a small amount of liquid needed to take the morning dose of medication.
1. Ensure in the morning (before UAE) that the patient is fasting.

2. Ensure that the patient took the medications for own chronic diseases.

3. Note that fact in the medical record.

8. Order the patient the following medications: 1 tablet of anxiolytic (for example, Estazolam) orally, 1 dose of intravenous antibiotics, 1 globule of Metronidazole intravaginally, Diclofenac $100 \mathrm{mg}$ rectal suppository.

1. Give the above medicines about $30-40$ min prior to UAE.

2. Note that fact in the medical record.

9. Prepare the operative field by ensuring that the left armpit and right groin are shaved.

1. If the above areas of skin are not shaved, shave them gently.

2. Note that fact in the medical record

10. Ensure that the patient does not have an intrauterine device (IUD).

1. If the patient has an IUD in the uterus, inform her of the increased risk of infection in the uterine cavity and offer to remove it before UAE.

2. If the patient does not agree to remove the IUD, note that fact in the informed consent of the patient or the medical record.

11. Ensure that the patient reports any allergic reactions to medicines, contrast agents, and disinfectants and note that fact and results in the medical record. 


\section{Performing the UAE procedure}

NOTE: This is addressed to interventional radiologists and anesthesiologists.

1. Perform the procedure in sterile conditions.

1. Put the patient on the operating table and disinfect the right inguinal region widely using a hospital disinfectant (with appropriate approval).

2. Glue the surgical drape around the surgical site (right groin)

2. Select the type of anesthesia: local or epidural anesthesia (EA) and note the patient's choice in the medical record.

3. UAE under local anesthesia.

1. Anesthetize (subcutaneous administration) $2 \%$ lignocaine solution at the site of the surgery.

2. Administer $5 \mathrm{mg}$ of morphine intravenously as needed.

3. Before starting the UAE procedure, make sure that the local anesthesia is operational.

4. UAE under EA

1. Prepare the patient properly for the EA.

2. Perform the EA before gluing the surgical drape.

3. Ensure that the patient qualified for the EA procedure does not have the following contraindications EA: procedure refusal, coagulopathy, thrombocytopenia, hemolytic disease, taking anticoagulants, shock, infection at the site of epidural injection, bacteremia, local anesthetics allergy, anatomical deformities of the spine, increased intracranial pressure, neurological disease, severe aortic or mitral stenosis ${ }^{94}$. Note that fact in the medical record.
4. Call an anesthesiologist and ask for EA.

5. Place a thin catheter to the epidural space in the lumbar region and give an anesthetic (procedure addressed for anesthesiologists).

6. Before starting the UAE procedure, make sure that the EA is operational.

5. The UAE procedure

1. Perform the UAE procedure in Angio Suit under fluoroscopy guidance.

2. Obtain access to the vascular system using the Seldinger technique ${ }^{95,96}$ (Figure 7, Figure 8, and Figure 9).

3. Insert a pigtail catheter into the abdominal aorta just below the renal arteries.

4. Perform angiography to visualize the vessels.

5. Perform aortonephrography to assess the anatomy of the vessels that supply the fibroids and to plan the procedure (Figure 10).

6. Perform angiography of the internal iliac artery in anterior-posterior and oblique projection to reveal the uterine artery ostium.

1. Start from the left side, then the right one, due to the site of a puncture and specific shape of the catheter. Depending on the caliber of the vessel, the main catheter or microcatheter is selectively introduced into the uterine artery (Figure 11).

7. Place the catheter deep in the uterine artery. Embolize the vessel with hydrogel particles. Due to the presence of uterine-ovary anastomosis, the size of which is estimated at approximately $500 \mu \mathrm{m}$, the 
suggested size of the particles for embolization is 700

$\mu \mathrm{m}$ to reduce the risk of "non-target embolization".

8. Continue embolization until the blood flow in the vessel is completely blocked. The endpoint of treatment is contrast stasis in the vessel, which proves its effective closure.

9. Perform the embolization of the uterine artery on the opposite side with the same access. The treatment lasts for about 0.5 to $1.0 \mathrm{~h}^{66,67}$ (Figure 12).

10. Assess the effectiveness of the embolization from a catheter placed in the internal iliac artery during control angiography. The absence of an active inflow of shading blood (to the uterine arteries) indicates that the procedure is considered technically correct (Figure 13).

11. Carefully remove the catheter.

12. Close the puncture site through manual compression with pressure dressing, which should be maintained for the next $6 \mathrm{~h}$ or closure device (Figure 14).

13. Describe the course of the UAE in the medical record.

\section{Patient care after the UAE procedure}

NOTE: This is addressed to gynecologists or vascular surgeons and anesthesiologists.

1. Start analgesic treatment: patient controlled analgesia (PCA) pump or EA.

1. Inform and discuss the options of analgesic treatment with the patient.

2. Inform the patient that EA provides better pain control and faster rehabilitation, compared to the PCA pump $^{76}$.
3. Note that fact and the patient's choice in the medical record.

2. Analgesic treatment of post-embolization pain using a PCA pump.

1. Prepare the syringe for the PCA pump with the morphine solution.

1. Fill the $50 \mathrm{~mL}$ syringe for the PCA pump with $50 \mathrm{mg}$ of morphine and solution of $0.9 \% \mathrm{NaCl}$ (concentration $1 \mathrm{mg} / \mathrm{mL}$ ).

2. Prepare the PCA pump for intravenous use.

1. Join the drain to the syringe.

2. Insert the venipuncture using a cannula.

3. Insert the syringe into the PCA pump.

4. Fill the drain, launch the PCA pump.

5. Turn on the START button.

6. Lock the PCA pump.

3. Set the PCA pump parameters (listed below) (Figure 15).

WARNING! Higher doses of Morphine may cause respiratory depression.

1. Use a Morphine concentration of $1 \mathrm{mg} / \mathrm{mL}$. Use a dose of morphine infusion on-demand (intravenous bolus) (Bolus p.) of $0.5 \mathrm{mg}$ with a time interval after which the next on-demand infusion can be provided (Lockout interval/ [Karencja]) of $5 \mathrm{~min}$ (to avoid respiratory depression). Use a time of intravenous morphine injection during on-demand infusion of $10 \mathrm{~s}$.

4. Ensure that all the above steps are done.

5. Order morphine in the PCA pump for up to $24 \mathrm{~h}$. 
1. Monitor pain according to the Numerical Rating Scale (NRS). Note the NRS value in the medical record.

2. Use the Pain Assessment Card (PAC).

6. Inform the patient that when the pain symptoms come, she should press the "joy-stick" herself, thereby obtaining the medicine on demand.

1. Ensure that the patient understands the instructions.

2. Observe the amount of on-demand "intravenous bolus" to "empty bolus" (launched during lockout interval - section 4.2.3 of the Patient care after the UAE PROTOCOL) (Figure 16).

3. If the ratio of "empty bolus" to on-demand "intravenous bolus" reaches a value higher than $2 / 1(67 \%$ / 33\%), use the procedure below (Figure

\section{7, Figure 18).}

4. To improve the effectiveness of pain relief therapy using a PCA pump, and reduce the risk of morphine side effects, consider using paracetamol or non-steroidal anti-inflammatory drugs in intravenous injections and standard doses.

7. Take care of the situations when the patient needs to leave the bed.

1. Do not turn off the pump.

2. Disconnect the drain and secure it with a stopper.

3. When the patient returns, reconnect the drain

3. Analgesic treatment of post-embolization pain using EA.

1. Continue EA if it was used for the UAE procedure.
2. If local anesthesia was used for the UAE procedure and the patient orders EA, before epidural catheter placement, ensure that $\mathrm{CBC}$ and coagulation tests are correct.

3. Ensure that the patient qualified for the EA procedure does not have the contradictions described in section 3.4.3 of Performing the UAE procedure. Note that fact in the medical record.

4. Call an anesthesiologist and ask for EA.

5. Make sure that the EA is operational.

6. Determine the EA work mode (bolus or continuous) with the anesthesiologist. Note that fact in the medical record.

1. In continuous mode (CEA), consult with an anesthesiologist and check the infusion pump settings.

2. In bolus mode (BEA), discuss with the anesthesiologist the time intervals between administering the local anesthetic agent.

7. Maintain the catheter for $24-48 \mathrm{~h}$, depending on the needs.

1. Monitor the pain with the NRS scale. Note the NRS value in the medical record.

2. Use the PAC.

3. Continue CEA/BEA until needed, no longer than $48 \mathrm{~h}$.

8. Continue analgesic treatment after PCA or EA if necessary.

1. Order Paracetamol $3 \times 1.0 \mathrm{~g}$ intravenously.

2. Order Diclofenac 2 x $50 \mathrm{mg}$ rectal suppository. 
9. If the above analgesic treatment is not enough, include supportive analgesics.

1. Order Ketoprofen $2 \times 100 \mathrm{mg}$ intravenously.

10. Treat urinary tract symptoms by ordering Furazidin 3 x $100 \mathrm{mg}$ orally.

\section{Control visit after the UAE procedure}

NOTE: This is addressed to gynecologists.

1. Perform the first control examination on the first day after UAE.

1. Assess the general condition of the patient.

2. Measure the patient's body temperature.

3. Rate pain after UAE according to the NRS scale. Use the PAC

4. Assess the wound where the vascular catheter was inserted.

5. Perform $\mathrm{CBC}$ and coagulation tests.

6. Perform a TVUS to assess the condition of the pelvic organs after UAE.

7. Perform a 3D TVUS using volume probe to assess the volume and vascularization indexes of the fibroid (VI, FI, and VFI).

2. If there are no complications, and the post-embolization pain has been controlled by oral analgesics, discharge the patient home.

1. Inform the patient that if fever, abdominal pain, and purulent vaginal discharge occur after hospitalization, the patient should immediately seek medical attention.

3. Perform the next control visit 3 months after UAE.
1. Perform a gynecological examination, $C B C$ test, and $\mathrm{AMH}$ or FSH serum level.

2. Perform MRI or TVUS to assess the condition of the pelvic organs after UAE (Figure 19).

3. Perform 3D TVUS using volume probe to assess the volume and vascularization indexes of the fibroid (VI, $\mathrm{FI}$, and $\mathrm{VFI}$ ).

4. Perform the last control visit 6 months after the UAE.

1. Perform a gynecological examination, $C B C$ test, and AMH or FSH serum level.

2. Perform a TVUS to assess the condition of the pelvic organs after UAE.

3. Perform 3D TVUS using volume probe to assess the volume and vascularization indexes of the fibroid (VI, $\mathrm{FI}$, and $\mathrm{VFI}$ ).

5. Assess the effectiveness of UAE.

1. Compare the results of the $\mathrm{CBC}$ test before and after UAE.

2. Compare the volumes and vascularization indexes of the fibroid (VI, FI, and VFI) before and after UAE.

6. Compare serum $\mathrm{AMH}$ or $\mathrm{FSH}$ levels before and after UAE to assess the possibility of iatrogenic damage to the ovaries during UAE as a result of "non-target" embolization.

7. Inform the patient that after uterine artery embolization, she should undergo regular gynecological check-ups.

8. In selected cases (no reduction of fibroid's symptoms, suspected tissue demarcation, or doubt in other additional examinations), perform an MRI one year after UAE (Figure 20). 


\section{Representative Results}

557 UAE procedures were performed in the period from 2009 to 2019. A mean of the patients' age was 38 years (3153 years of age). Technical success was achieved in 547 patients $(98.2 \%)$.

The mean reduction of the fibroid volume (MRI volume assessment) 3 months after UAE procedure performed in the period from 2009 to 2013 in the group of 206 patients aged 32 to 52 years (mean age: 39 years) was $62 \%$. The smallest reduction was $9 \%$ (patient with hyalinized fibroid). Complete reduction (100\%) was achieved in patients with the separated submucosal fibroid (FIGO 0). $90 \%$ of patients reported satisfaction after the UAE procedure ${ }^{64}$.

The mean reduction of the fibroid volume 3 months after UAE (ultrasound VOCAL volume assessment) in a group of 65 patients aged 29-52 years (mean age: 43.1 years) was $50.1 \%(2.7 \%-93.5 \%)$. Before the UAE procedure, median fibroid volume was $101 \mathrm{~cm}^{3}$ (range from 23.6 to 610.0 $\mathrm{cm}^{3}$ ), whereas, after 3 months, the reduction of median fibroid volume to $50.4 \mathrm{~cm}^{3}$ (range from 6.9 to $193.9 \mathrm{~cm}^{3}$ ) was observed. The Spearman correlation test showed a statistically significant, but relatively weak, positive correlation $(R=0.33 ; p=0.006)$ between the initial dominant fibroid volume and percentage volume reduction. Interestingly, smaller fibroids showed a great variability of fibroid volume reduction at 3 months after UAE, while larger fibroids showed a stable, predictable reaction to $\mathrm{UAE}^{72}$.

Reduction of doppler vascular indices (VI, $\mathrm{FI}$, and $\mathrm{VFI})$ in the group of 17 patients 3 months after UAE was observed. The percentage reduction in $\mathrm{VI}$ and $\mathrm{VFI}$ was $95.4 \%$, whereas in $\mathrm{FI}$ the reduction was $58.3 \%{ }^{75}$.
Assessment of ovarian reserve was performed in 30 patients aged 33-40 years (mean age: 35 years) 3 months after UAE. The mean dominant fibroid volume was $107.75 \mathrm{~cm}^{3}$ (range from 87.4 to $131.1 \mathrm{~cm}^{3}$ ). The following markers of the ovarian reserve were investigated: antral follicle count (AFC), $A M H$, inhibin $B$ (INHB), FSH, and estradiol (E2). A significant decrease in AFC (56.7\%; $p<0.001)$, AHM (36.7\%; $p<0.001)$, INHB (46.7\%; $p<0.001)$, and E2 (43.3\%; $p<$ 0.001 ) was observed. Simultaneously, a significant increase in $\mathrm{FSH}$ serum level $(43.4 \% ; \mathrm{p}<0.001)$ was observed ${ }^{65}$.

Three months after UAE procedure, in two patients with submucosal fibroids (FIGO 0 ) (with diameters of $6 \mathrm{~cm}$ and $8 \mathrm{~cm}$ ) uterine inversion was observed during excretion of demarked, necrotic fragments of fibroids through the cervical canal, which resulted in an emergency hysterectomy.

Post-embolization pain reduction (according to PAC) with the use of PCA (procedure 4.2 of the Patient care after the UAE Protocol) was assessed in 60 patients on the NRS scale on the day after the UAE procedure. The median NRS immediately after UAE was 10 (range 5-10), while after treatment the median NRS was rated 4 (range 1-5). The Spearman's correlation test between the initial volume of fibroids (median $194.5 \mathrm{~cm}^{3}$, range $79-411 \mathrm{~cm}^{3}$ ) and NRS immediately after UAE showed a statistically significant, strong positive correlation ( $R=0.6 ; p<0.001)$, whereas the correlation between the initial volume of fibroids and NRS after treatment showed a statistically significant, weak positive correlation $(R=0.34 ; p<0.001)$. Analyzing the above relationships, it can be concluded that major fibroids after UAE cause stronger post-embolization pain after UAE. However, the treatment of post-embolization pain after UAE of smaller fibroids with the use of PCA gives better results. 


\section{jove}

Summarized data for Representative Results are provided in

\section{Table 1.}

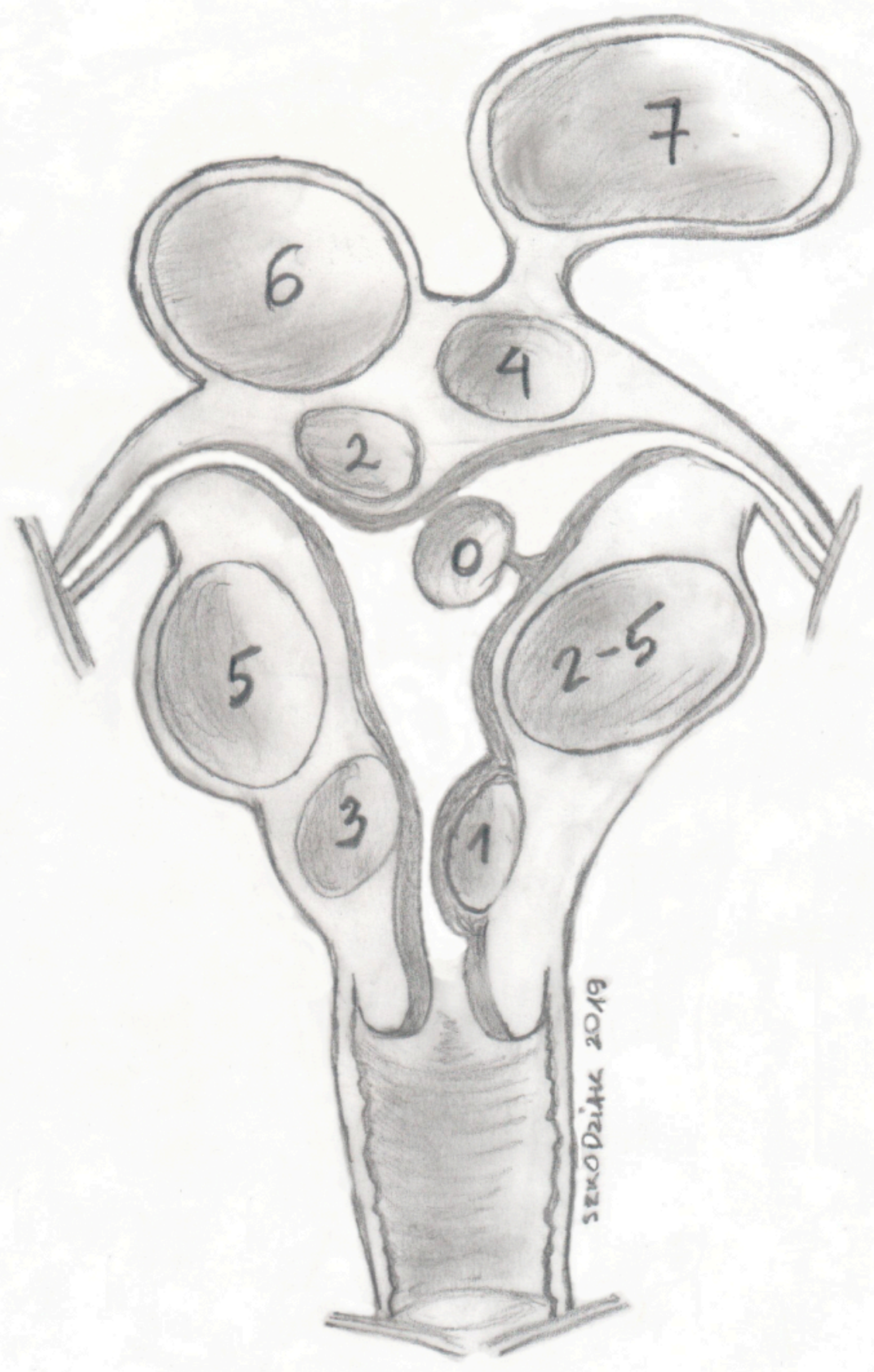

Figure 1: FIGO uterine fibroids classification. Please click here to view a larger version of this figure. 


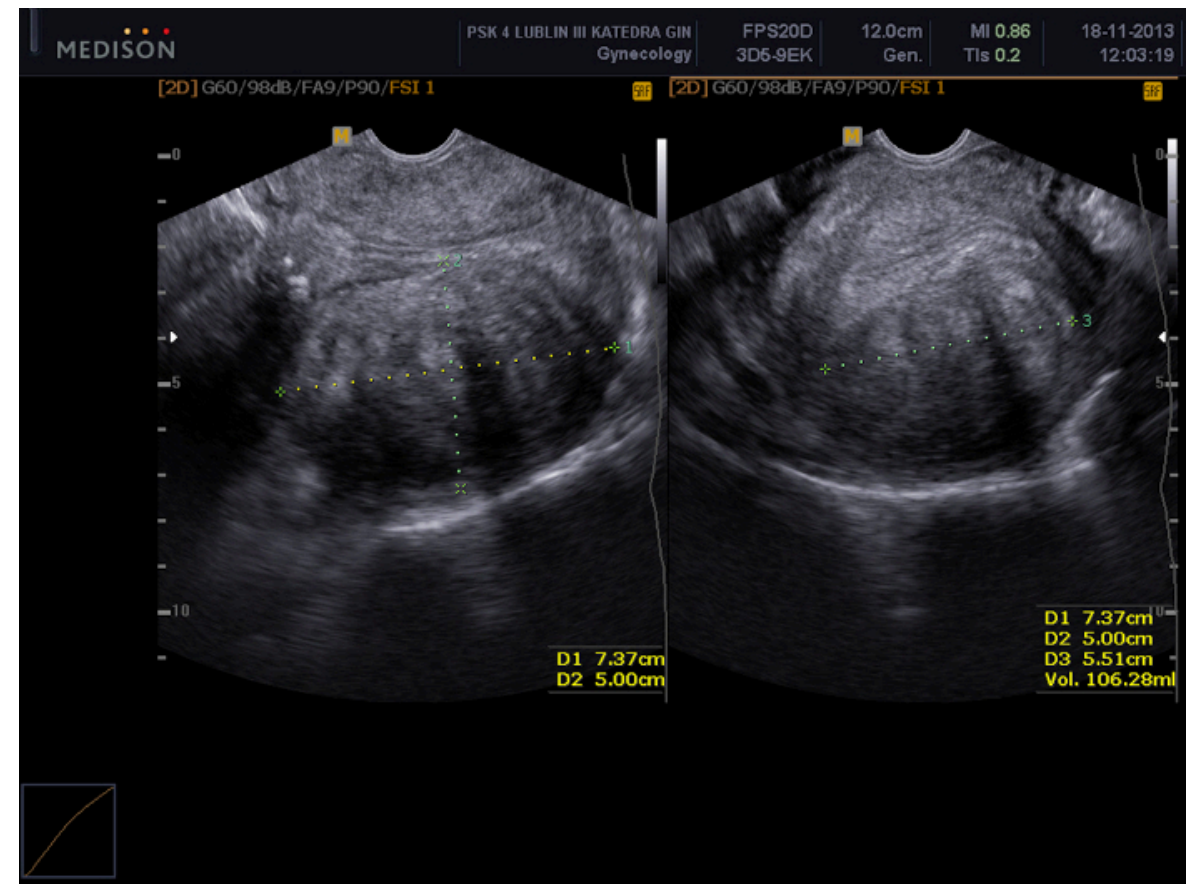

Figure 2: Pelvic examination using a transvaginal ultrasound scan.

Visible a uterine fibroid (FIGO 5) with dimensions of $73 \times 50 \times 55 \mathrm{~mm}$. Please click here to view a larger version of this figure. 


\section{jove}

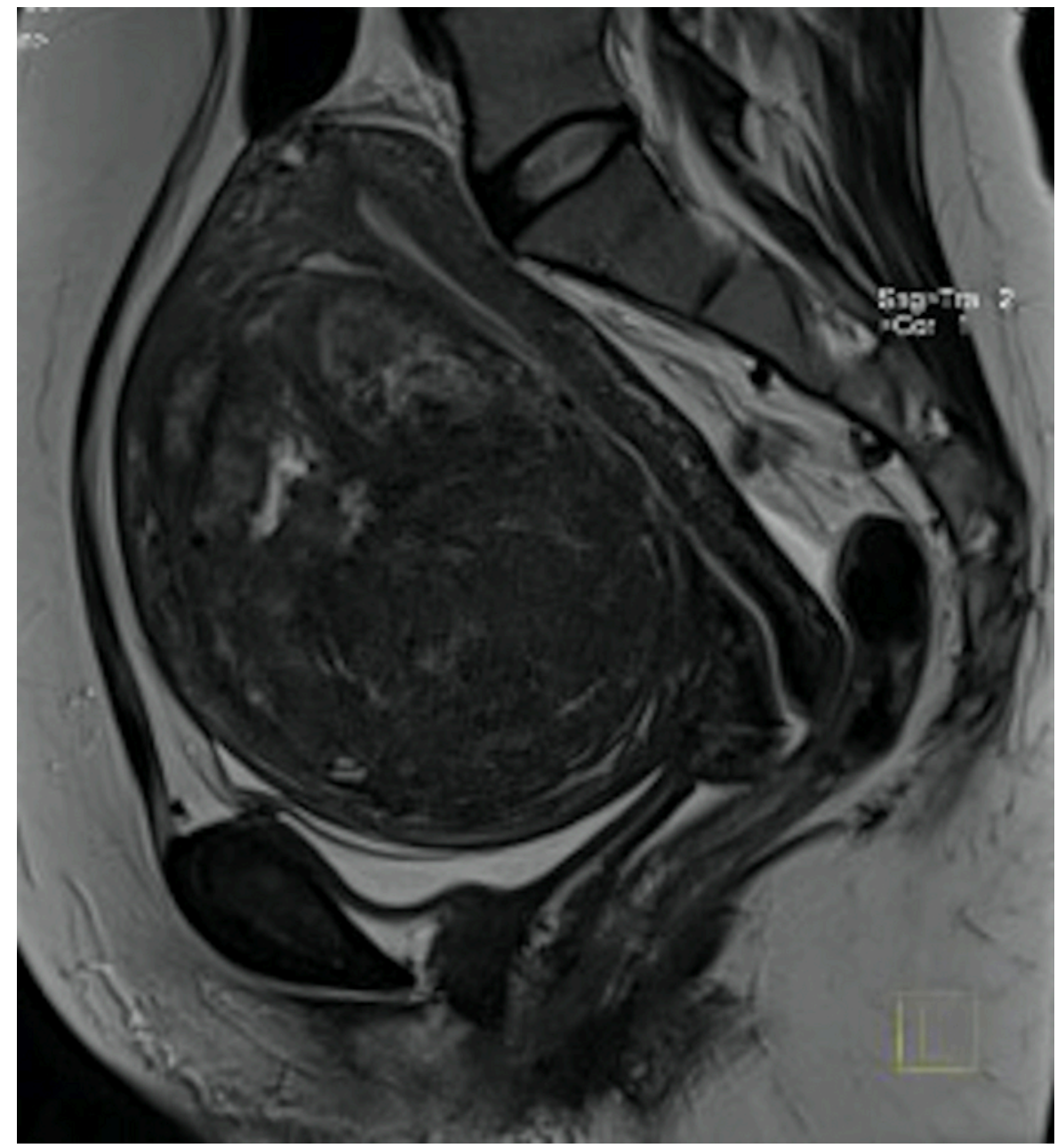

Figure 3: Pelvic MRI examination in pre-qualification for UAE.

Visible in the sagittal section a big uterine fibroid (FIGO 2-5) with mass effect. Please click here to view a larger version of this figure. 


\section{jove}

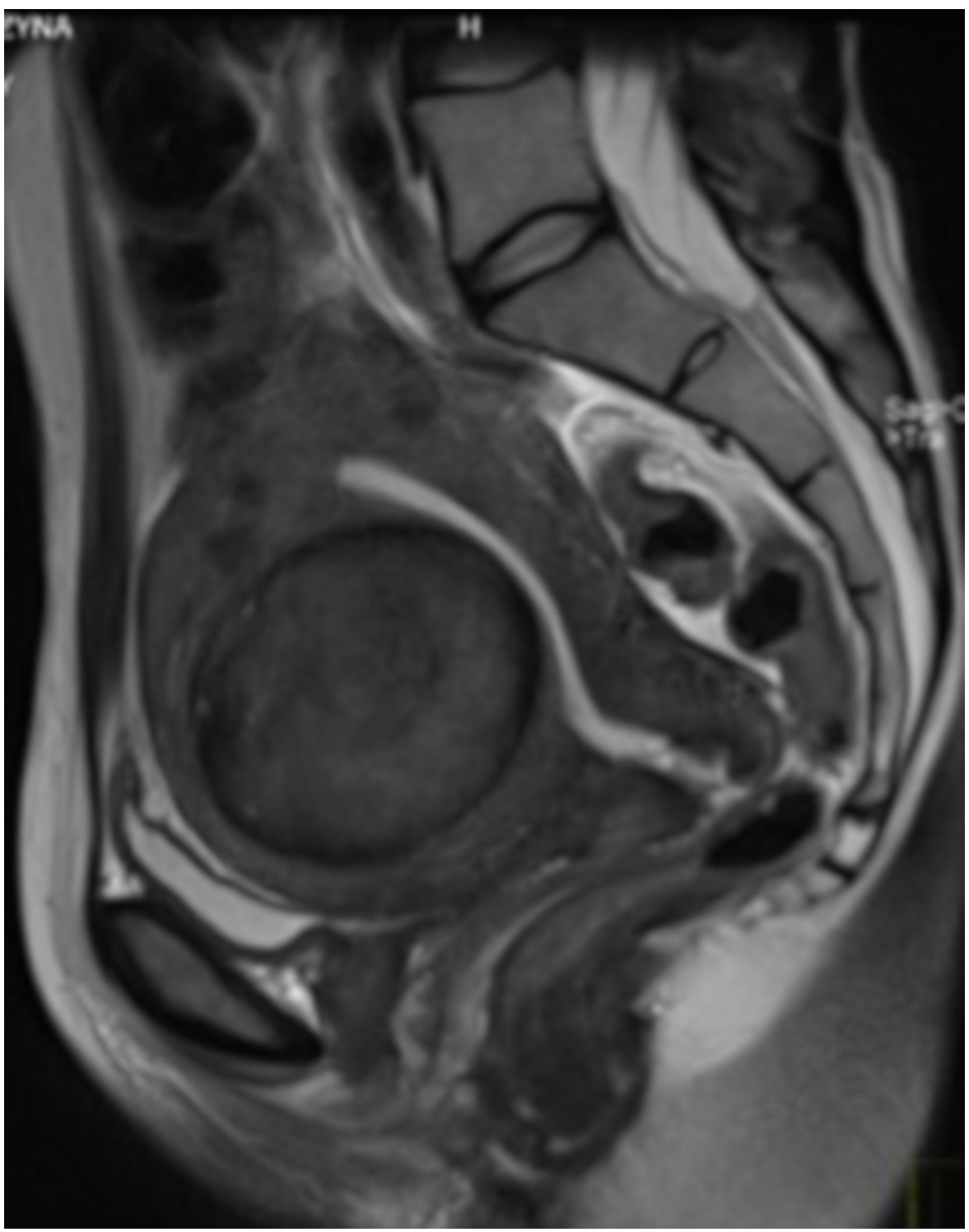

Figure 4: Pelvic MRI examination in pre-qualification for UAE.

Visible in the sagittal section a uterine fibroid (FIGO 2-5). Please click here to view a larger version of this figure. 


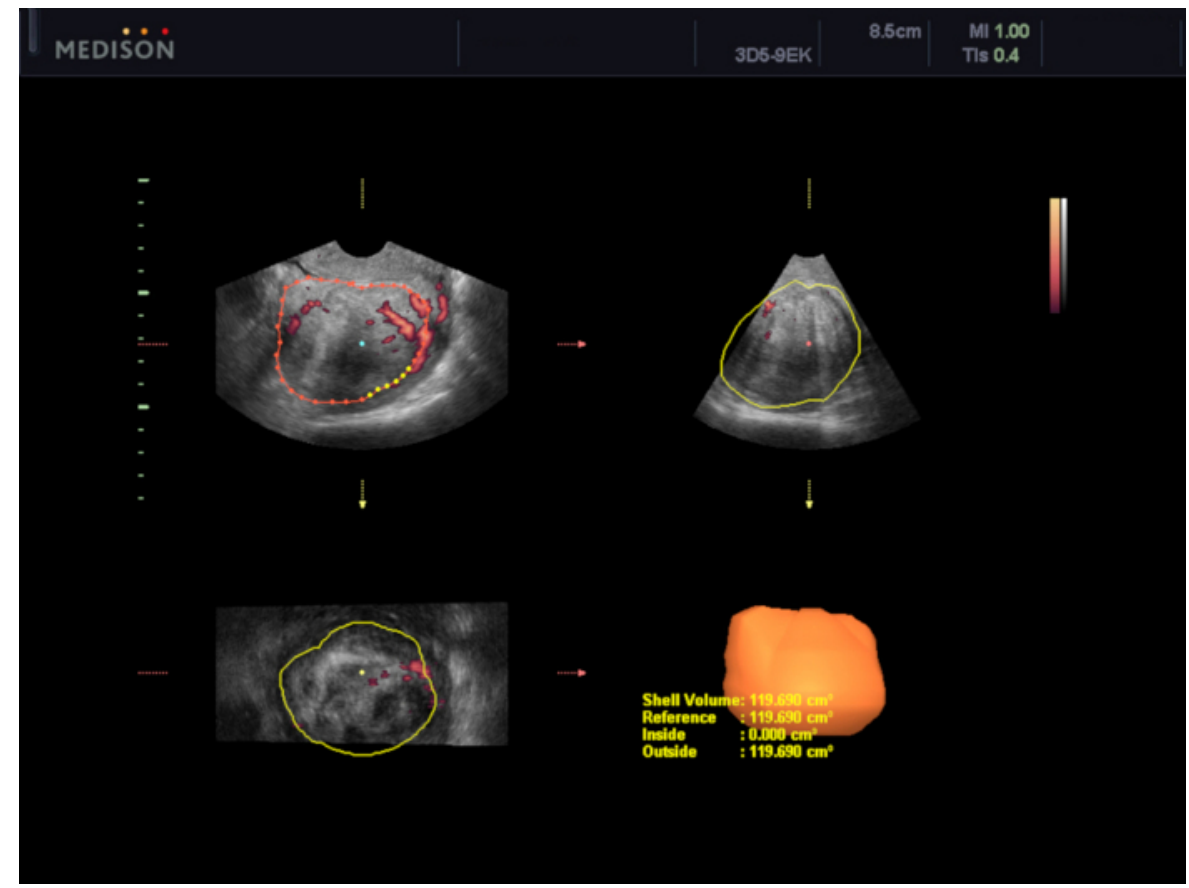

Figure 5: Assessment of uterine fibroid's volume using VOCAL software.

In this case, the volume is estimated at $119.7 \mathrm{~cm}^{3}$. Please click here to view a larger version of this figure. 


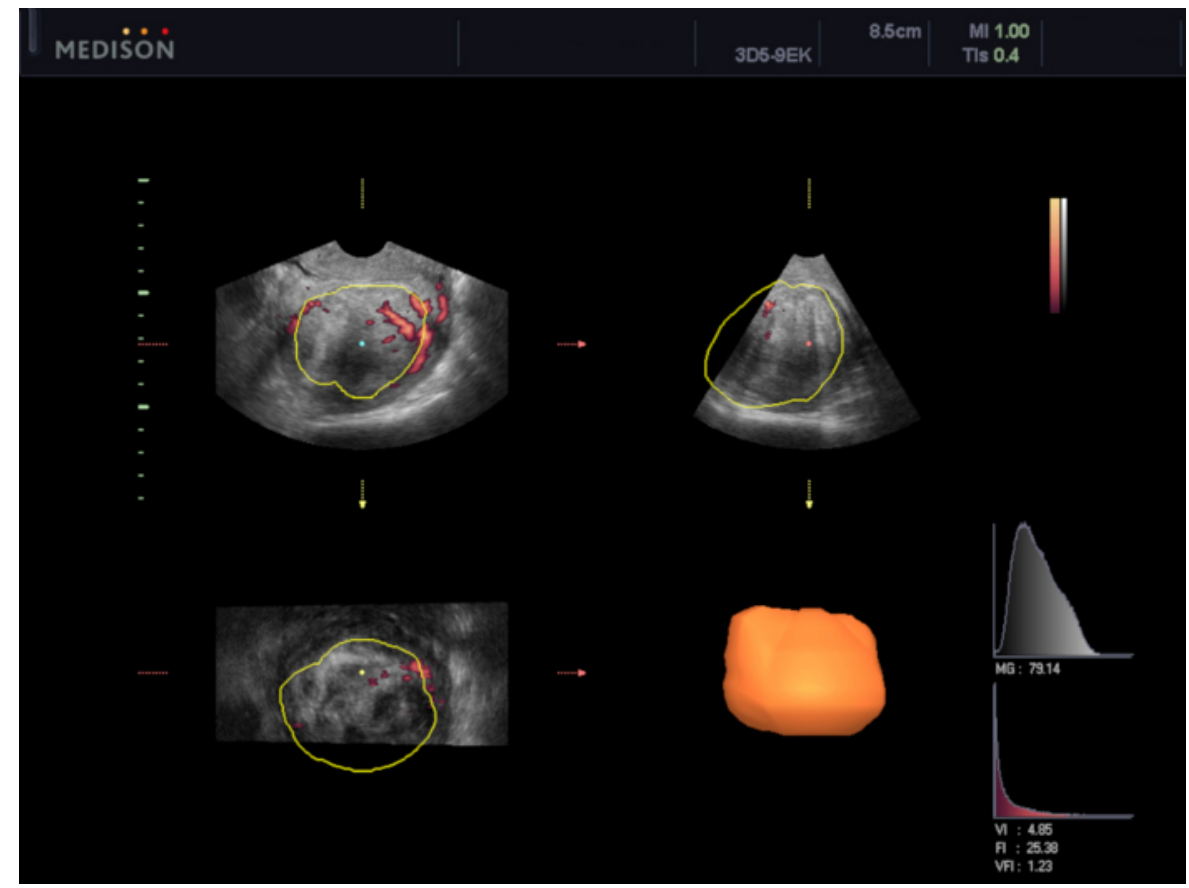

Figure 6: Assessment of uterine fibroid's vascularization using VOCAL software.

In this case, the vascularization indexes were calculated (VI 4.85, FI 25.38, and VFI 1.23). Please click here to view a larger version of this figure. 


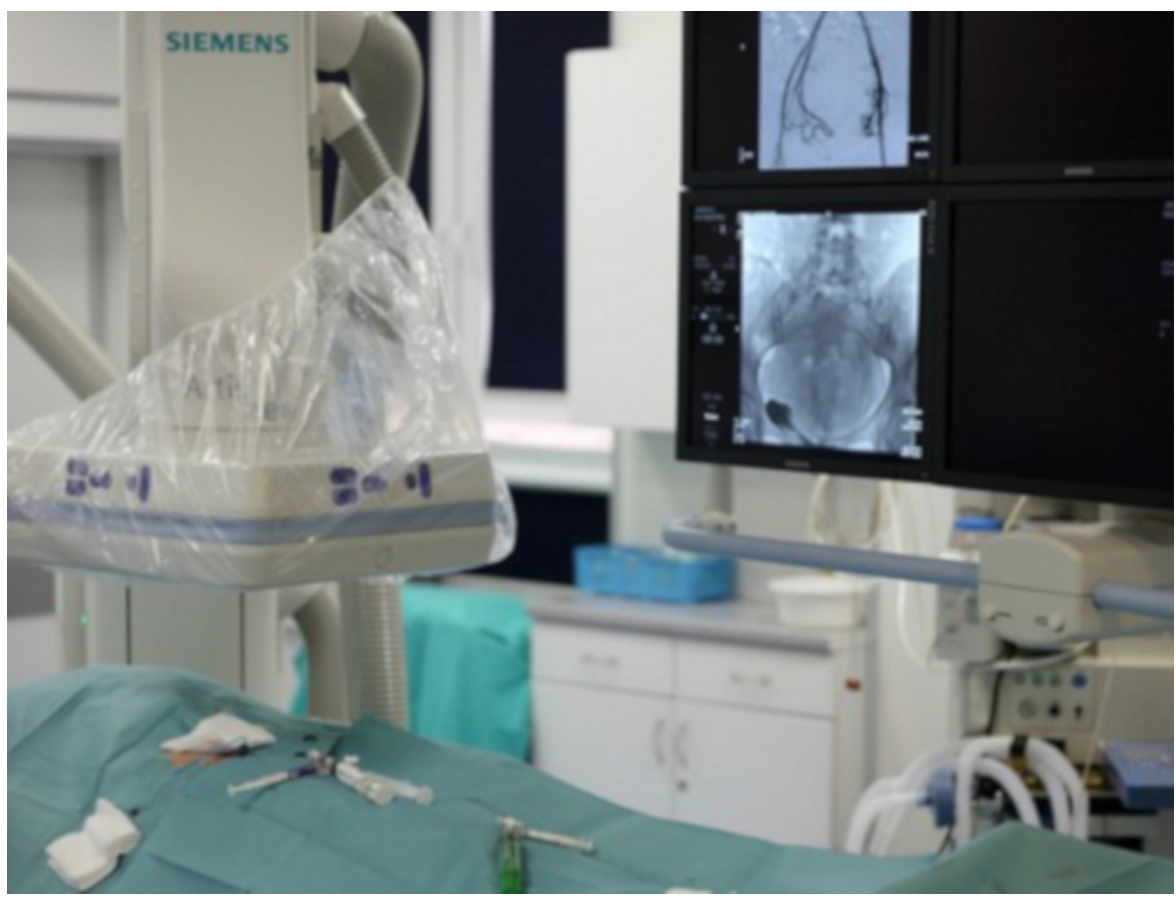

Figure 7: The picture shows a fragment of the angiographic laboratory.

In the lower-left corner, the patient with an exposed groin through which more tools are introduced. In the upper-left corner, the $\mathrm{C}$-arm of the angiograph can be seen. In the upper-right corner, the monitors are visible, where the operator tracks the input tool. Please click here to view a larger version of this figure. 


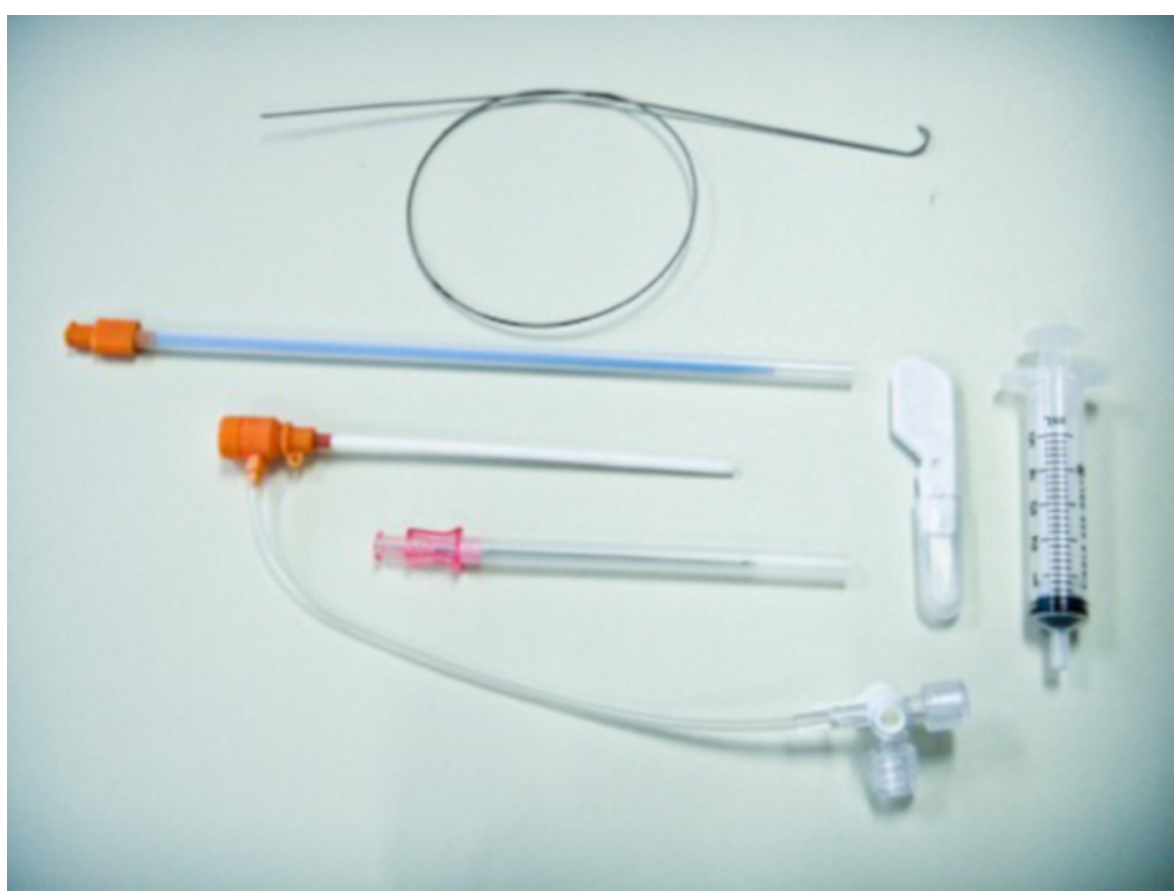

Figure 8: A set for arterial punctures.

From the bottom: a needle, a vascular lock with an introducer, and a guide. Please click here to view a larger version of this figure. 


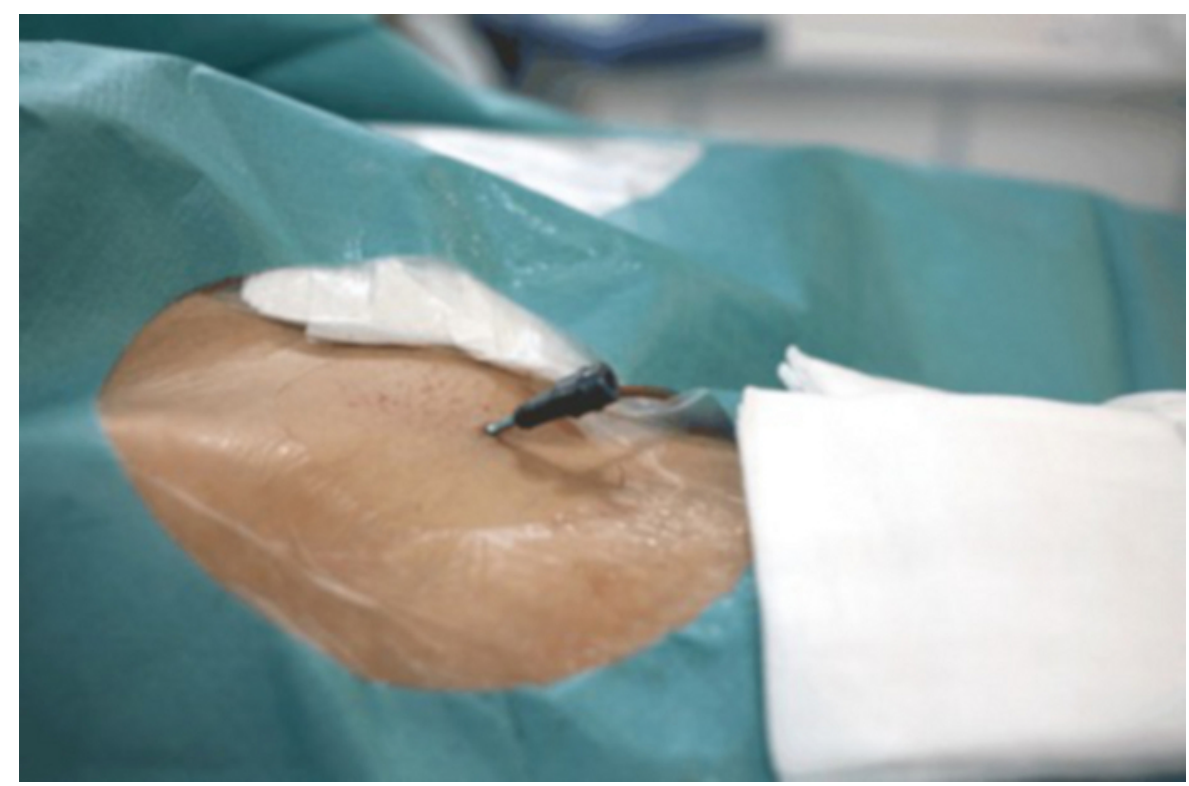

Figure 9: Close-up of the groin with visible vascular lock inserted into the femoral artery. Please click here to view a larger version of this figure.

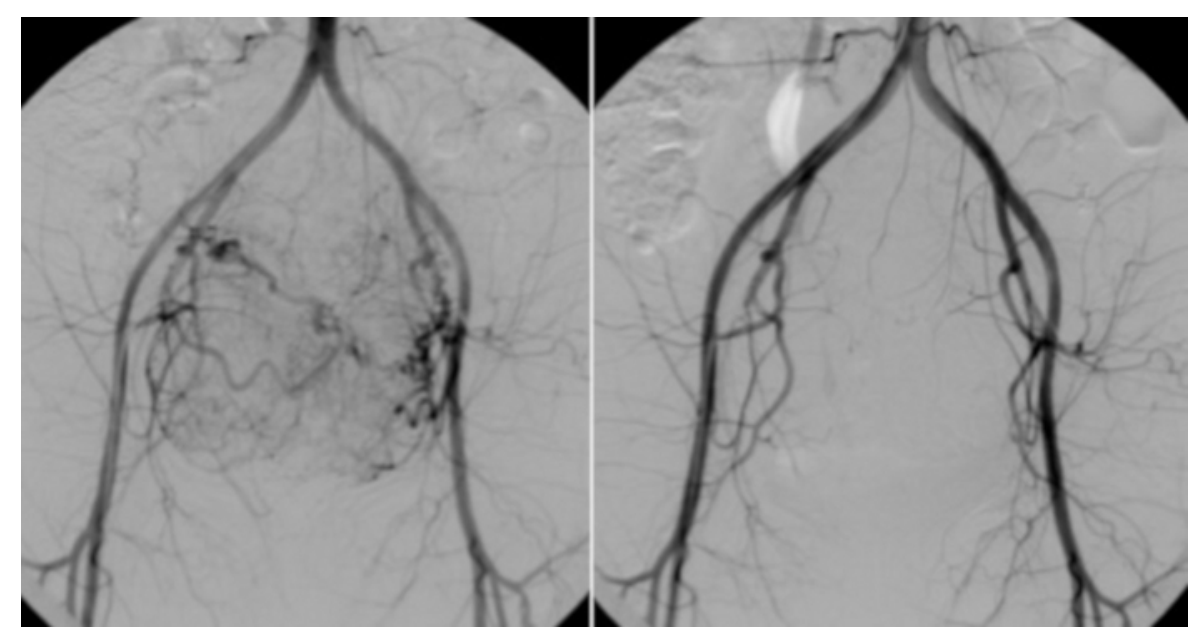

Figure 10: On the left, angiography from a catheter placed in the abdominal aorta.

The visible vascular bearing of uterine myomas. For comparison (on the right), a control test performed after UAE. Please click here to view a larger version of this figure. 


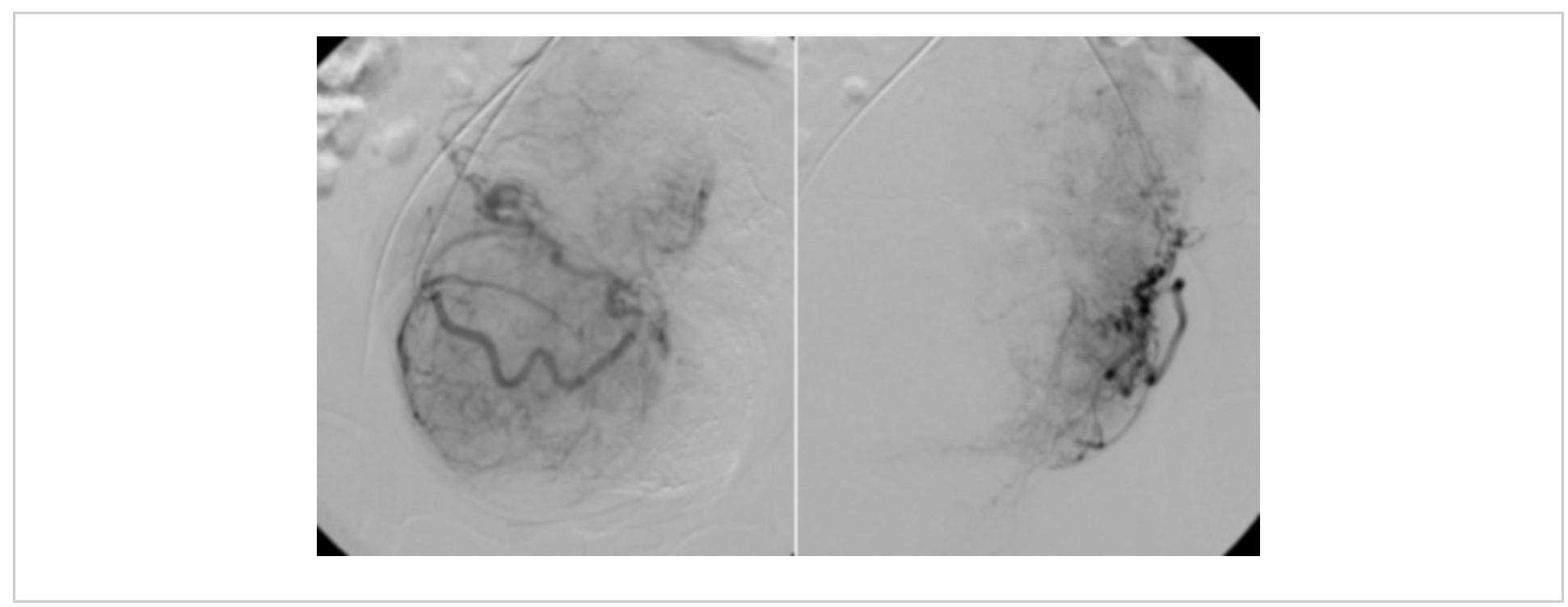

Figure 11: Selective angiography using RUC catheter placed in the proximal parts of the uterine arteries.

Visible vascular bed of uterine fibroids. Please click here to view a larger version of this figure. 


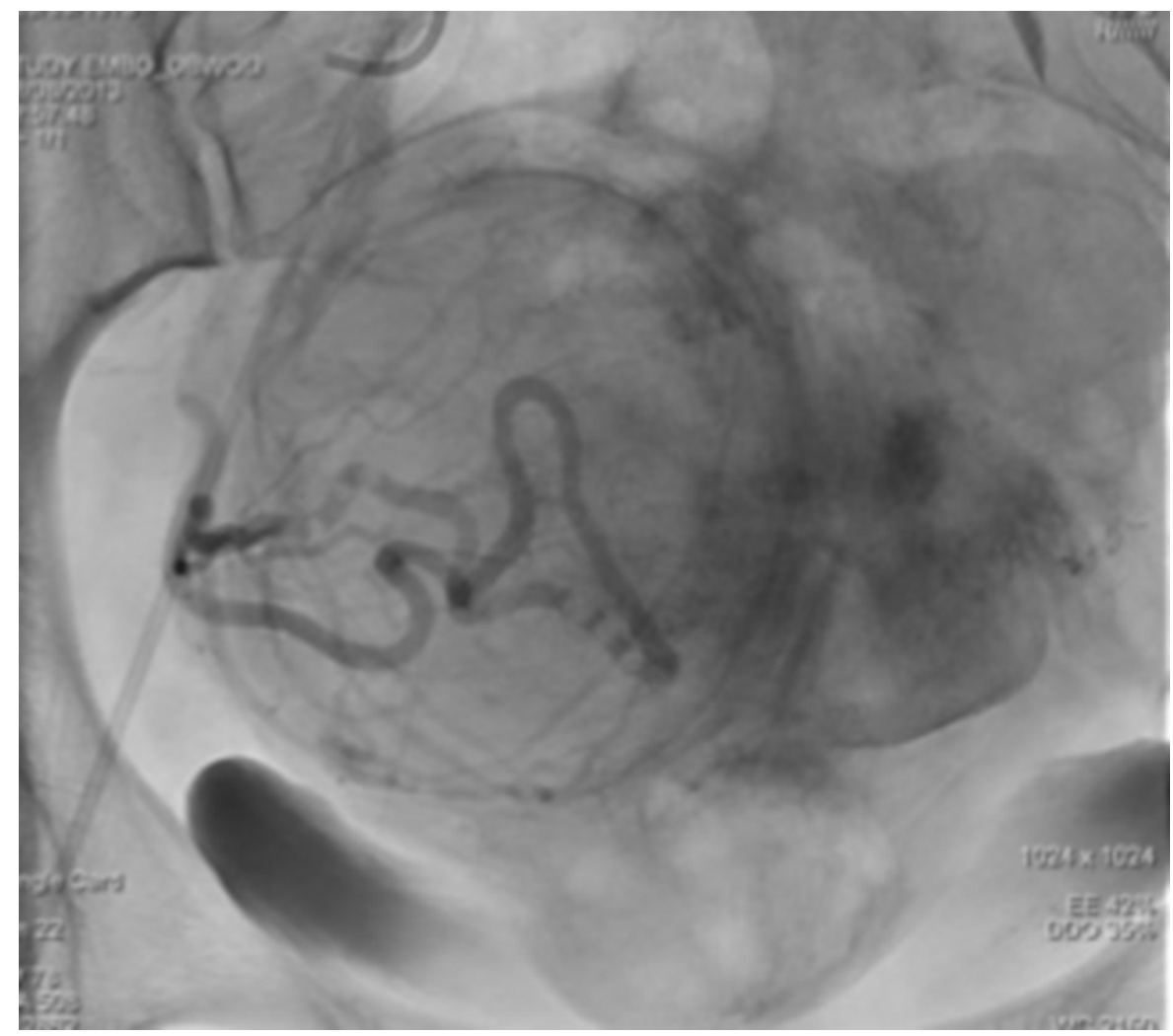

Figure 12: The single X-ray imaging showing the stasis of the contrast agent in the right uterine artery. Please click here to view a larger version of this figure. 

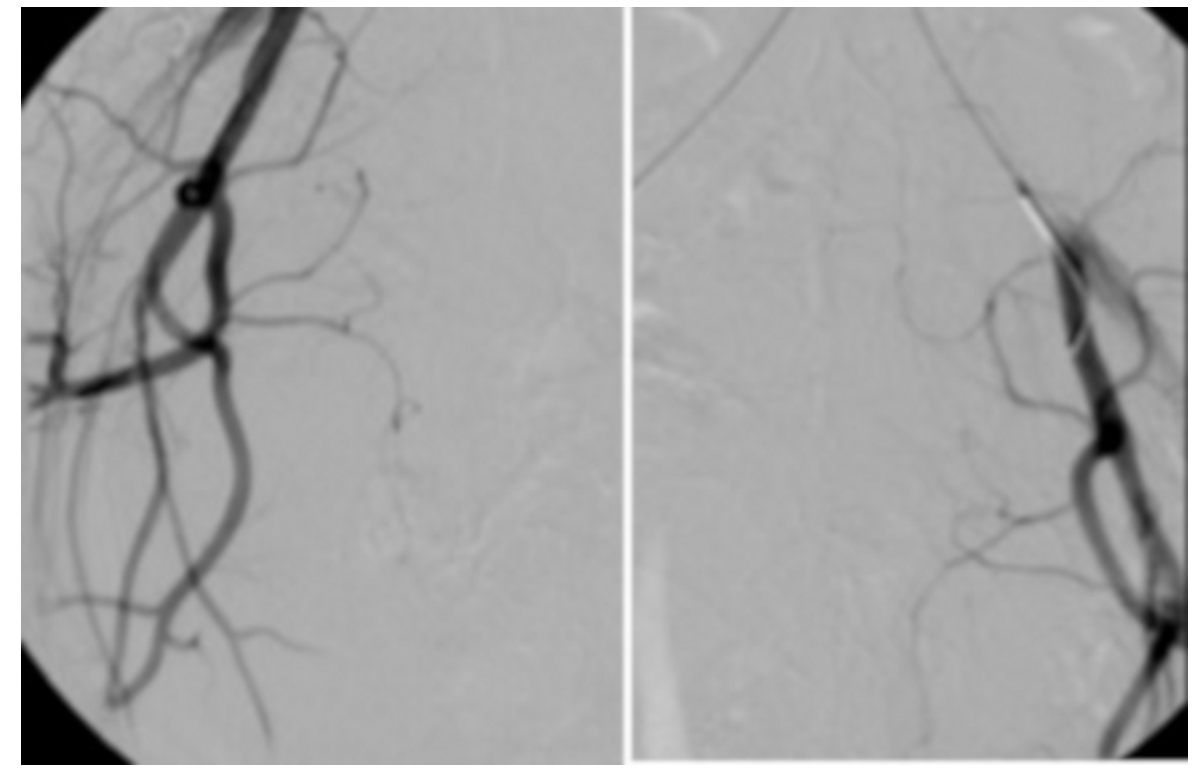

Figure 13: Control angiography performed from the main catheter located, respectively, in the left and right iliac artery, confirms the lack of inflow of fresh blood (shading) into the uterine arteries. Please click here to view a larger version of this figure.

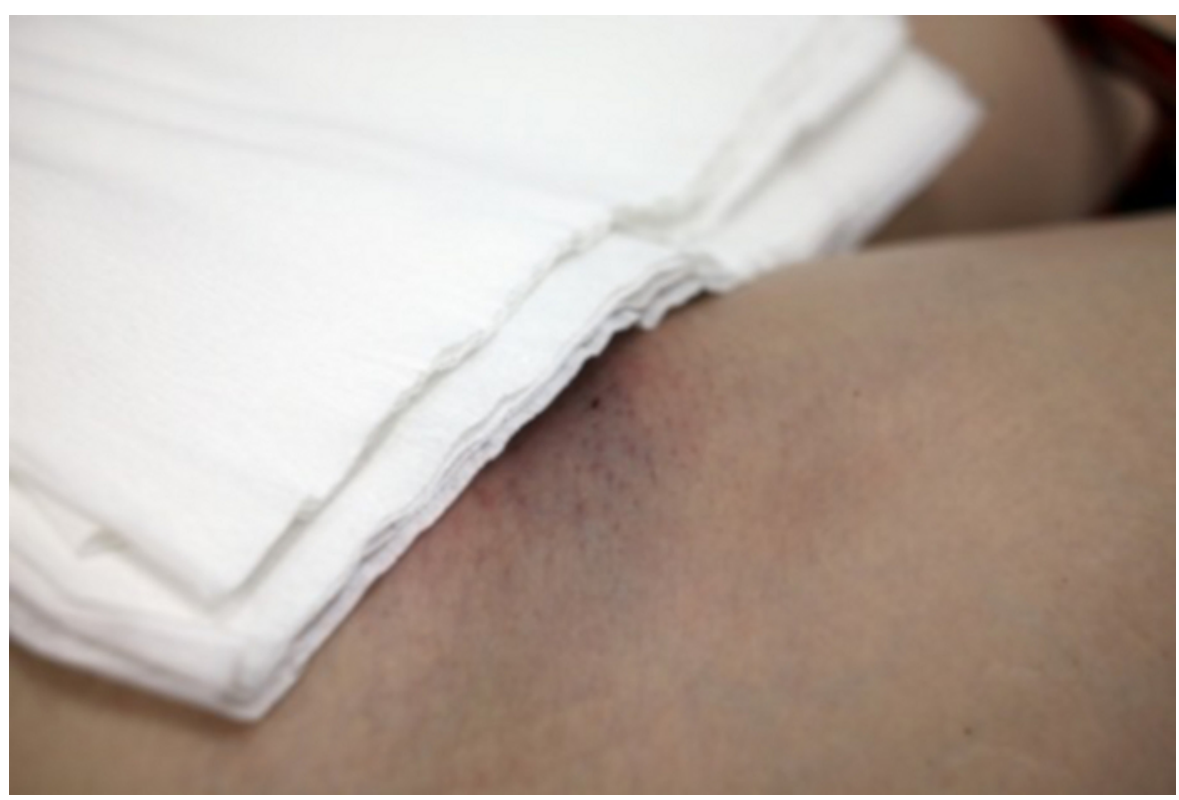

Figure 14: The vascular access site after the completion of UAE.

Visible $2 \mathrm{~mm}$ incision near the right groin. Please click here to view a larger version of this figure. 


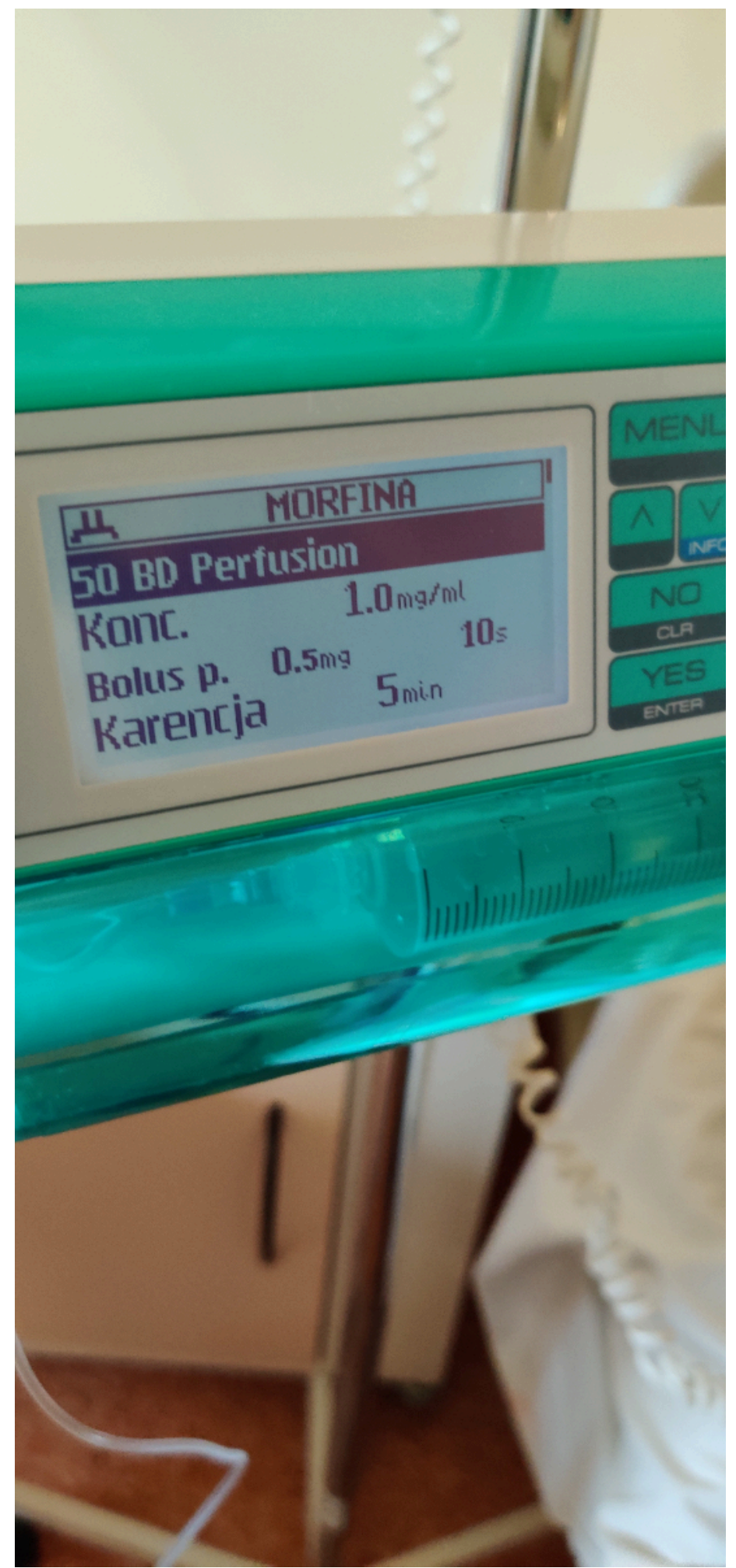

Figure 15: PCA pump parameter settings for post-embolization pain after UAE treatment (section 4.2.3 of the Patient care after the UAE PROTOCOL). Please click here to view a larger version of this figure. 


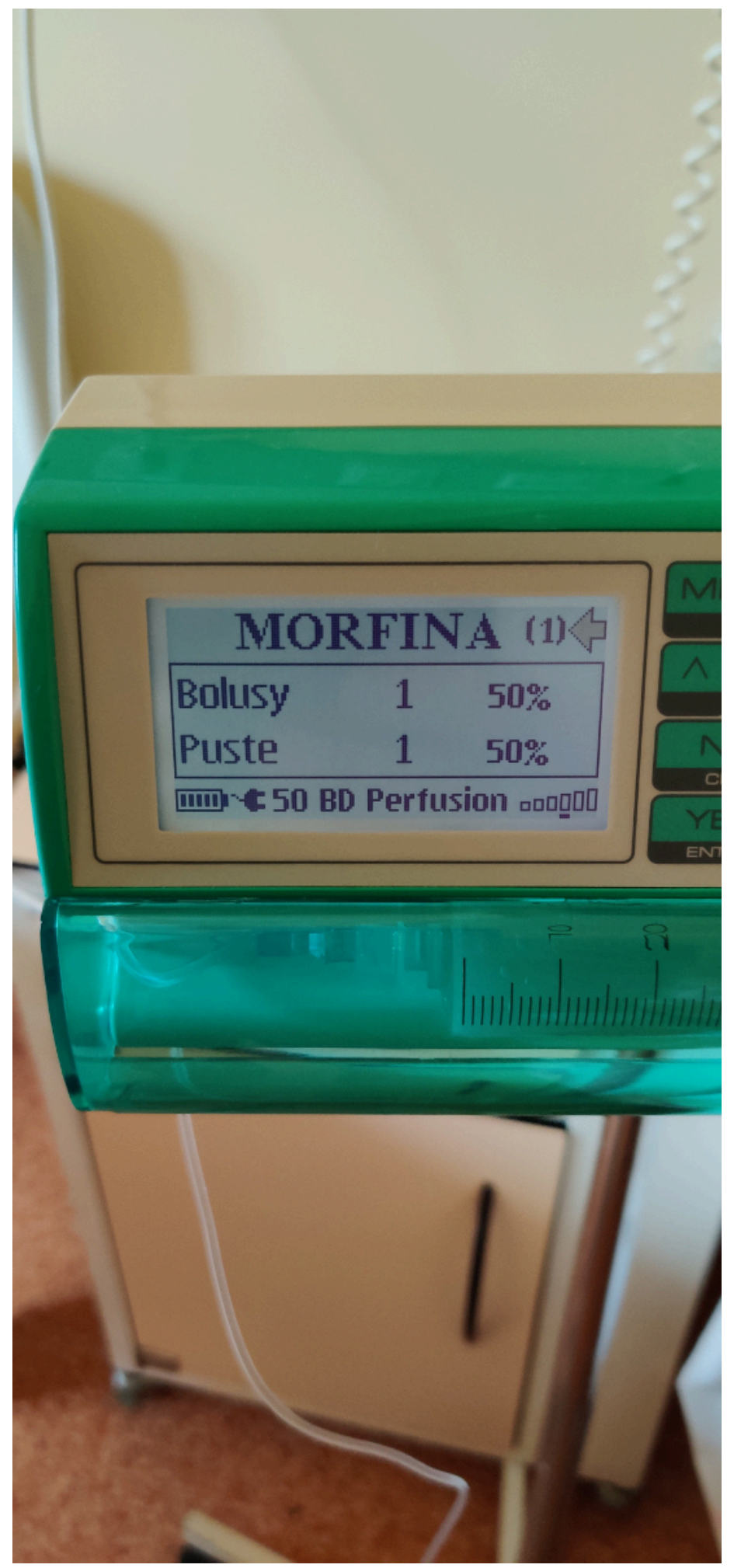

Figure 16: PCA pump in operation. 
The ratio of on-demand intravenous bolus to "empty bolus" (section 4.2.6.2. of the Patient care after the UAE PROTOCOL) 1:1 (50\%:50\%). Please click here to view a larger version of this figure.

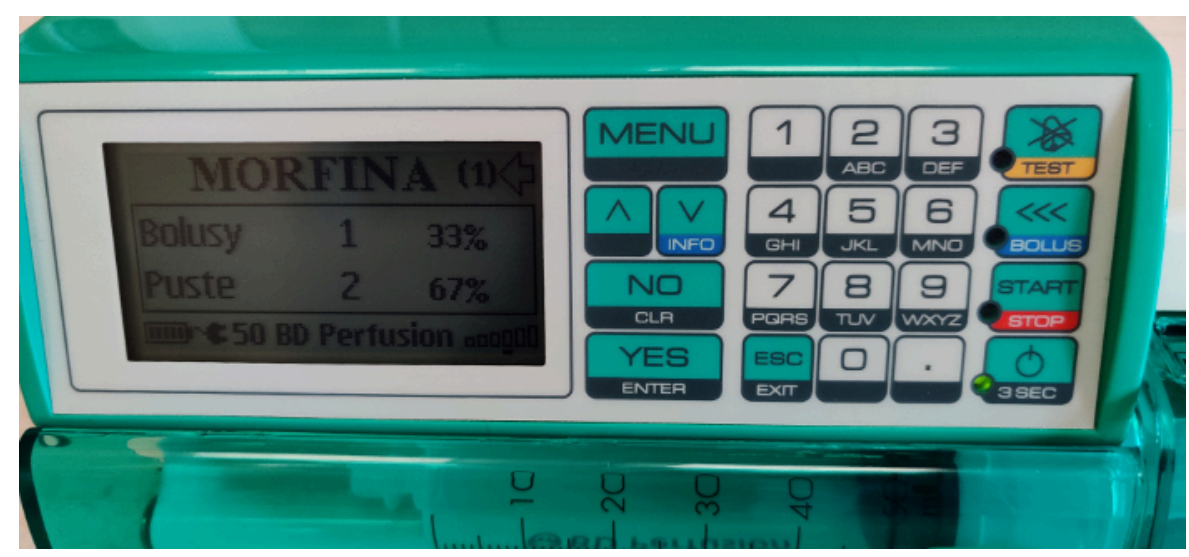

\section{Figure 17: PCA pump in operation.}

The ratio of on-demand intravenous bolus to "empty bolus" (section 4.2.6.3. of the Patient care after the UAE PROTOCOL) 1:2 (33\%:67\%). This requires additional analgesic treatment (procedure 4.2.6.4. of the Patient care after the UAE PROTOCOL). Please click here to view a larger version of this figure.

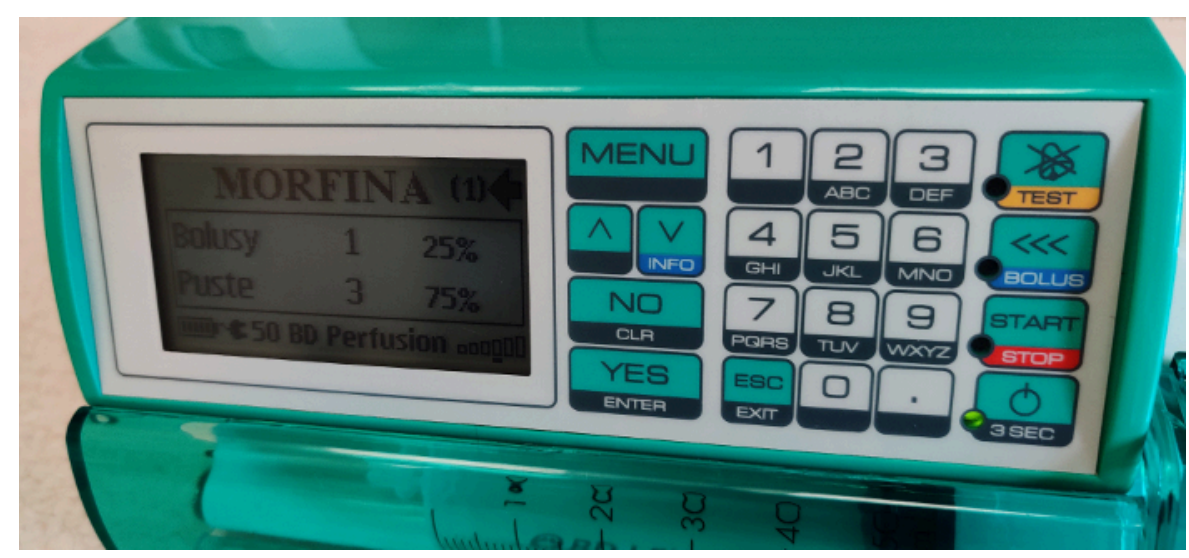

\section{Figure 18: PCA pump in operation.}

The ratio of on-demand intravenous bolus to "empty bolus" (section 4.2.6.3. of the Patient care after the UAE PROTOCOL) 1:3 (25\%:75\%). This requires continuing additional analgesic treatment (procedure 4.2.6.4. of the Patient care after the UAE PROTOCOL). Please click here to view a larger version of this figure. 


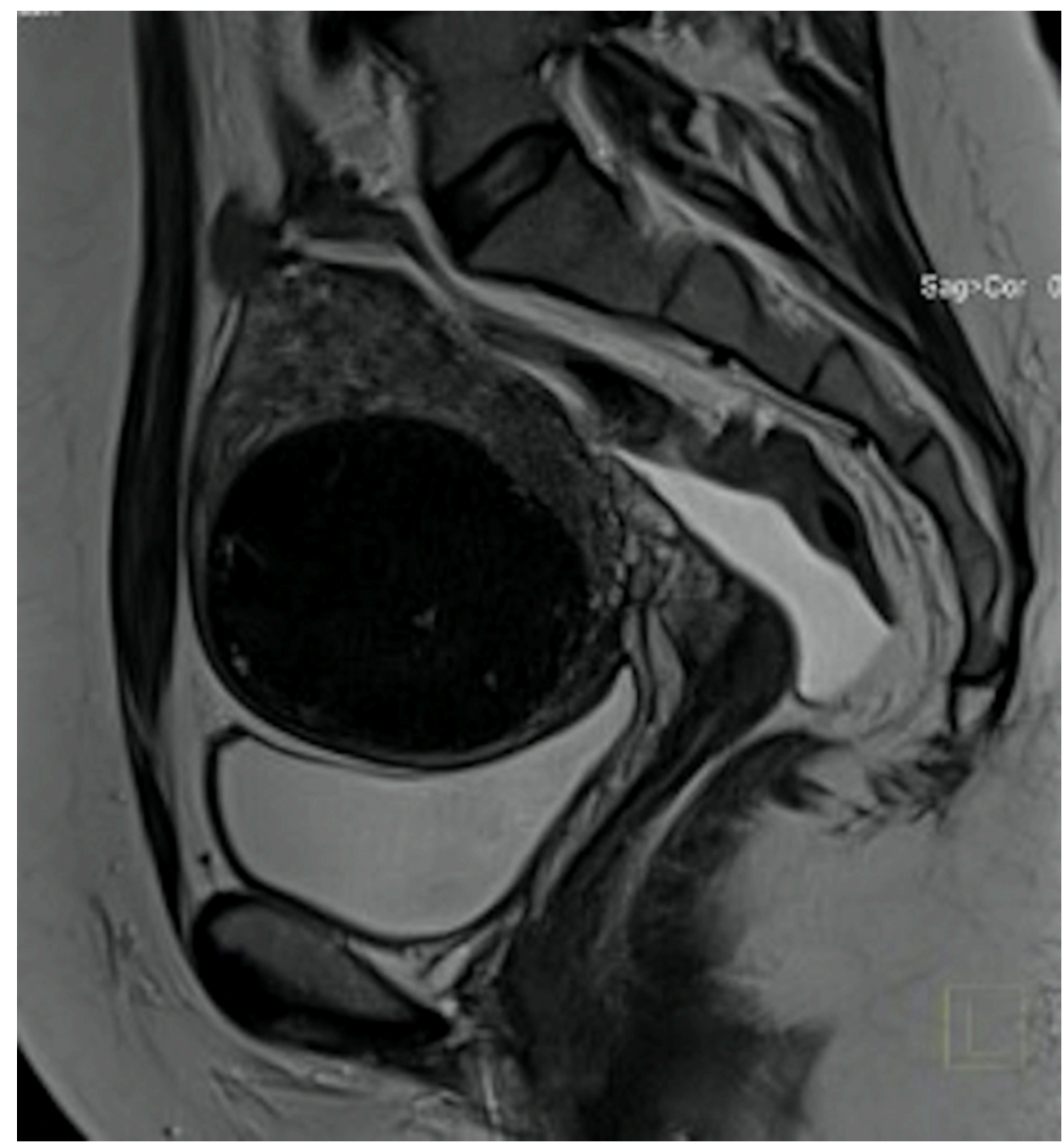

Figure 19: Pelvic MRI examination 3 months after the UAE procedure (the same case as in Figure 3).

Visible in the sagittal section is a uterine fibroid (FIGO 5), significantly smaller than before the procedure, different density of the fibroid tissue. Please click here to view a larger version of this figure. 


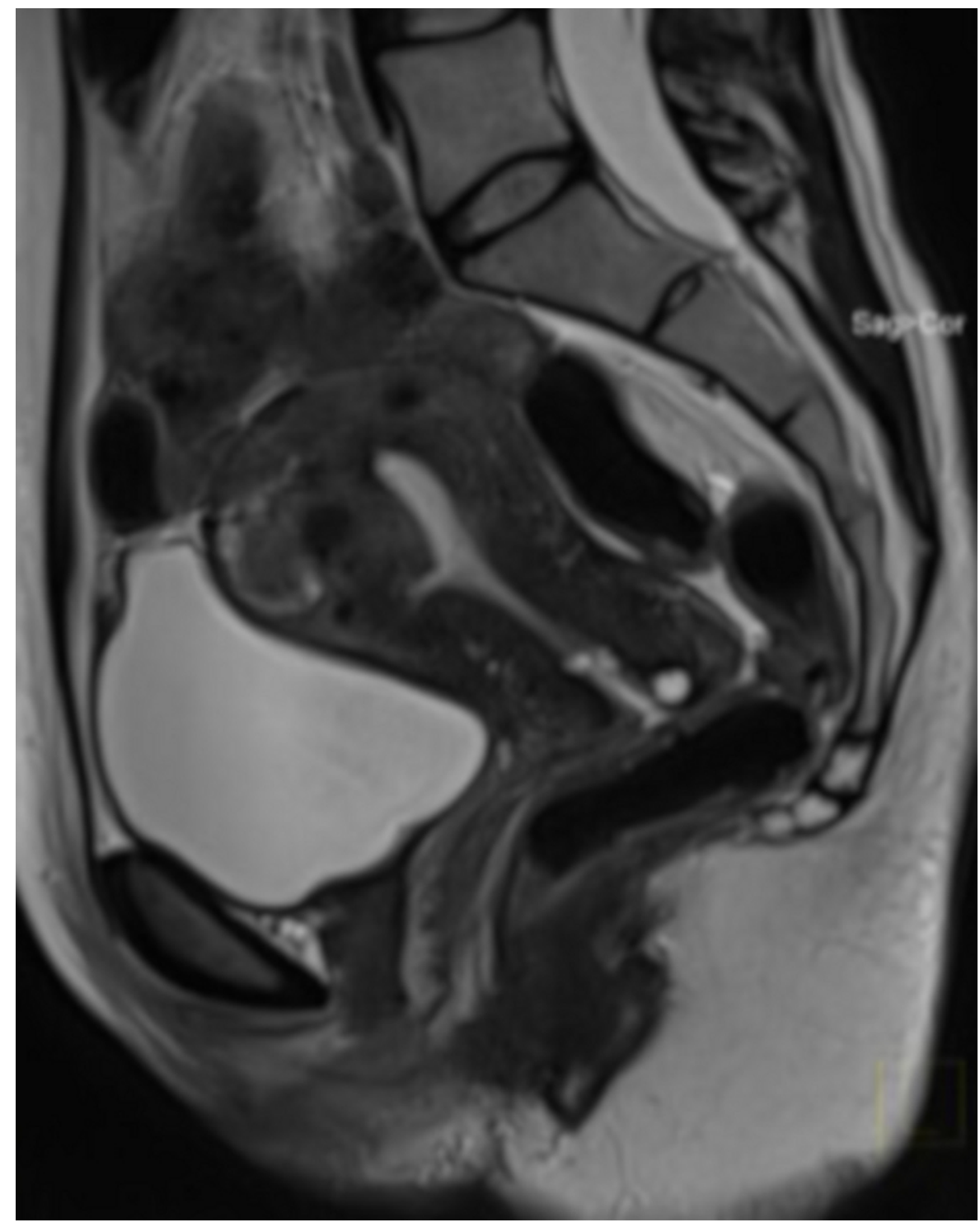

Figure 20: Pelvic MRI examination 1 year after UAE procedure (the same case as in Figure 4).

Visible in the sagittal section is a uterine fibroid (FIGO 2-5), with a very large volume reduction after UAE. MRI was performed due to suspected tissue demarcation after the procedure (no possibility to assess the fibroid structure in a bimanual examination). Please click here to view a larger version of this figure. 
REPRESENTIVE RESULTS / THE LUBLIN PROTOCOL $64,65,72$

\begin{tabular}{|c|c|c|}
\hline \multicolumn{2}{|c|}{ The number of UAE procedures performed in the period from 2009 to 2019} & 557 \\
\hline \multicolumn{2}{|c|}{ Technical success } & Achieved in in 547 patients (98.2\%) \\
\hline \multicolumn{2}{|c|}{$\begin{array}{l}\text { The mean reduction of the fibroid volume (MRI volume assessment) } 3 \text { months } \\
\text { after UAE in the group of } 206 \text { patients aged } 32 \text { to } 52 \text { years (mean age - } 39 \text { years) }\end{array}$} & $62.0 \%(9.0-100.0 \%)$ \\
\hline \multicolumn{2}{|c|}{$\begin{array}{c}\text { The mean reduction of the fibroid volume (ultrasound VOCAL } \\
\text { volume assessment) } 3 \text { months after UAE in the group of } \\
65 \text { patients aged } 29-52 \text { years (mean age }-43.1 \text { years) }\end{array}$} & $50.1 \%(2.7-93.5 \%)$ \\
\hline \multirow[t]{5}{*}{ Assessment of ovarian reserve } & Decrease in AFC & $56.7 \%(p<0.001)$ \\
\hline & Decrease in AMH & $36.7 \%(p<0.001)$ \\
\hline & Decrease in INHB & $46.7 \%(p<0.001)$ \\
\hline & Decrease in E2 & $43.3 \%(p<0.001)$ \\
\hline & Increase in FSH & $43.4 \%(p<0.001)$ \\
\hline \multirow{2}{*}{$\begin{array}{l}\text { Post-embolization pain reduction } \\
\text { (according to PAC) with the use of } \\
\text { PCA (procedure } 2 \text {. of the "Patient } \\
\text { care after the UAE" PROTOCOL) } \\
\text { assessed in the group of } 60 \text { patients }\end{array}$} & $\begin{array}{l}\text { The median NRS } \\
\text { immediately after UAE }\end{array}$ & 10 (range $5-10$ ) \\
\hline & The median NRS on the day after UAE & 4 (range $1-5$ ) \\
\hline
\end{tabular}

Table 1: Representative Results of UAE technique in the treatment of symptomatic uterine fibroids performed in accordance with Lublin Protocol.

\section{Discussion}

Because of the differences in the structure, size, localization, and symptoms of uterine fibroids, the creation of a uniform UAE protocol has not been an easy task. There have been many discrepancies regarding the assumptions of this therapeutic method with the expectations of patients, both at the stage of qualification and the effects of treatment. More than once, patients referred for UAE did not report any clinical signs of fibroids and were not aware that these uterine tumors would not be removed radically. The only explicit expectation was to get rid of fibroids without surgery.

Therefore, it is important that the patient understands the assumptions of this method, accepts it, and knows the differences regarding alternative methods of uterine fibroid treatment. Her conscious choice (section 1.1. of the Qualification for UAE Protocol) is a critical point, and its proper implementation will allow the protocol to be continued. 
During the implementation of the protocol, some procedures are repeated. This is intended and results from the formula adopted by this journal, in which individual commands are written in imperative mode and are directed to one person. However, several doctors are often involved in the qualification, preparation, and other UAE stages. These are also critical points of the protocol; their omission may result in UAE under non-optimal conditions or with the presence of contraindications. Hence the division of the protocol into 5 chapters. This allows it to be continued by various specialists, and the repeated protocol points are then independently checked.

An additional difficulty in creating a uniform protocol of uterine artery embolization in the treatment of uterine fibroids is the current large number of recommendations (as many as 11) that relate to the same procedure ${ }^{41}$. Although their assumptions are similar, as always, "the devil is in the detail", which details required unification. Relative contraindications regarding the location of fibroids or reproductive plans of patients undergoing $U A E$ are the most controversial during qualifications. Applying more stringent criteria proposed by SOGC, patients should be excluded from this procedure, while more liberal RCOG recommendations allow qualification for $U A E^{14,32,43}$. The question is what to do. During the protocol creation, we based the decision (apart from extensive literature) on the analysis of our cases and experience gained, which requires an individual approach to each patient. Therefore, the protocol does not exclude the performance of UAE in patients with relative contraindications (sections 1.4 of the qualification for UAE Protocol). The right qualification and preparation for UAE seem to be the key to therapeutic success. The technique itself is also very important, as well as UAE care, which ensures not only therapeutic success or patient satisfaction but also the lack of complications described above.

Regardless of the number of fibroids, all lesions are embolized during one procedure. Usually, more the fibroids, more will be the embolization material that will be injected. This extends the duration of the embolization but does not change the procedure. UAE can be modified if we see an obvious connection to the ovarian artery, which may result in an increased risk of non-target embolization. We can then close such a connection (e.g., using coils), thus separating the supply of the ovaries and the uterus, and then continue embolization with the use of $700 \mu \mathrm{m}$ particles. If it is not possible to implant coils, then we increase the particle diameter to $900 \mu \mathrm{m}$.

There are also some cases when the fibroids can be supplied from the side of the ovarian arteries; then, the uterine arteries are hypoplastic. In these cases, to successfully perform embolization, a microcatheter should be inserted into the ovarian artery and past the ovary, depositing the embolization material into the uterine vascular bed, while maintaining proper ovarian supply.

The undoubted advantage of embolization is the fact that it is not a technically difficult procedure and does not require sophisticated equipment.

The strong points of the protocol are the points regarding the treatment of post-embolization pain, which at least half of the patients had not heard about when qualifying for UAE. The standard procedure that we propose is the use of a PCA pump (section 4.2 of the Patient care after the UAE Protocol), and the results obtained confirm the high effectiveness of such a treatment. 
Regarding future modifications to the UAE protocol, it seems possible to change section 10 of the preparing for the UAE Protocol, which requires removal of the IUD from the uterus before the procedure due to the risk of inflammation and sepsis. In large follow-up studies, the risk of infection in the pelvis combined with the presence of an IUD is less than 1 in $1300^{49}$.

UAE has been treated as an experimental method since its first use because it required assessing the effectiveness and examining the complications that this procedure may give in short and long-term periods. During these years, indications and contraindications have been modified based on new test results and clinical observation. Current data, including several randomized controlled studies, recognize UAE as a valuable treatment method for symptomatic uterine fibroids, whose effectiveness and safety have been well established.

The creation of the above protocol is due to a thorough analysis of current literature, relevant recommendations and experience gained as a result of the close cooperation of gynecologists and surgical radiologists during the decade.

\section{Disclosures}

The authors have nothing to disclose.

\section{STATEMENT:}

Piotr Szkodziak as the author of Figure 1, illustrating the FIGO uterine fibroids classification, allows free use of the figure for scientific and educational applications without any modifications. The intention to modify the figure should be accepted by the author (piotr.szkodziak@gmail.com).
The authors would like to thank the entire team of the 3rd Chair and Department of Gynecology and Department of Interventional Radiology and Neuroradiology, Medical University of Lublin for their help in implementing the Uterine Arteries Embolization Lublin protocol.

\section{References}

1. Tinelli, A. et al. Myoma pseudocapsule: A distinct endocrino-anatomical entity in gynecological surgery. Gynecological Endocrinology. 25 (10), 661-667 (2009).

2. Tinelli, A. et al. Myomas: anatomy and related issues. Minerva Ginecologica. 68 (3), 261-273, at <https:// pubmed.ncbi.nlm.nih.gov/26785282/> (2016).

3. Laganà, A. S. et al. Epigenetic and genetic landscape of uterine leiomyomas: a current view over a common gynecological disease. Archives of Gynecology and Obstetrics. 296 (5), 855-867 (2017).

4. Vanharanta, S. et al. Distinct expression profile in fumarate-hydratase-deficient uterine fibroids. Human Molecular Genetics. 15 (1), 97-103 (2006).

5. Hug, K. et al. Physical mapping of the uterine leiomyoma $t(12 ; 14)(q 13-15 ; q 24.1)$ breakpoint on chromosome 14 between SPTB and D14S77. Genes, Chromosomes \& Cancer. 11 (4), 263-266 (1994).

6. Ozisik, Y. Y. et al. Cytogenetic findings in a symplastic leiomyoma. Cancer Genetics and Cytogenetics. 67 (1), 79-80 (1993).

7. Ozisik, Y. Y., Meloni, A. M., Surti, U., Sandberg, A. A. Deletion 7q22 in uterine leiomyoma. A cytogenetic review. Cancer Genetics and Cytogenetics. 71 (1), 1-6 (1993).

\section{Acknowledgments}


8. Sparic, R., Mirkovic, L., Malvasi, A., Tinelli, A. Epidemiology of uterine myomas: A review. International Journal of Fertility and Sterility. 9 (4), 424-435 (2016).

9. Stewart, E. A. Uterine fibroids. Lancet. 357 (9252), 293298 (2001).

10. Williams, A. R. W. Uterine fibroids - what's new? F1000Research. 6, 2109 (2017).

11. Stewart, E. A. Uterine fibroids. The New England Journal of Medicine. 372 (17), 1646-1655 (2015).

12. Al-Hendy, A., Myers, E. R., Stewart, E. Uterine fibroids: burden and unmet medical need. Seminars in Reproductive Medicine. 35 (6), 473-480 (2017).

13. de la Cruz, M. S. D., Buchanan, E. M. Uterine fibroids: Diagnosis and treatment. American Family Physician. 95 (2), 100-107 (2017).

14. Vilos, G. A. et al. The management of uterine leiomyomas. Journal of Obstetrics and Gynaecology Canada. 37 (2), 157-178 (2015).

15. Stewart, E. A. Uterine fibroids. The New England Journal of Medicine. 372 (17), 1646-1655 (2015).

16. Sabry, M., Al-Hendy, A. Medical treatment of uterine leiomyoma. Reproductive sciences (Thousand Oaks, Calif.). 19 (4), 339-353 (2012).

17. Istre, O. Management of symptomatic fibroids: conservative surgical treatment modalities other than abdominal or laparoscopic myomectomy. Best Practice \& Research. Clinical Obstetrics \& Gynaecology. 22 (4), 735-747 (2008).

18. Donnez, J., Dolmans, M. M. Uterine fibroid management: from the present to the future. Human Reproduction Update. 22 (6), 665-686 (2016).
19. Rogers, T. S., Bieck, A. M. Management of Uterine Fibroids. American Family Physician. www.aafp.org/afp. 99 (5), at <www.aafp.org/afp/ahrq.> (2019).

20. Chwalisz, K., Taylor, H. Current and Emerging Medical Treatments for Uterine Fibroids. Seminars in Reproductive Medicine. 35 (6), 510-522 (2017).

21. Saridogan, E. Surgical treatment of fibroids in heavy menstrual bleeding. Women's Health (London, England). 12 (1), 53-62 (2016)

22. Fritton, K., Borahay, M. A. New and Emerging Therapies for Uterine Fibroids. Seminars in Reproductive Medicine. 35 (6), 549-559 (2017).

23. Ciavattini, A. et al. Hypovitaminosis D and "small burden" uterine fibroids: Opportunity for a Vitamin D supplementation. Medicine (Baltimore). 95 (52) (2016).

24. Friend, D. R. Drug delivery for the treatment of endometriosis and uterine fibroids. Drug Delivery and Translational Research. 7 (6), 829-839 (2017).

25. Donnez, J., Courtoy, G. E., Dolmans, M. M. Fibroid management in premenopausal women. Climacteric: The Journal of the International Menopause Society. 22 (1), 27-33 (2019).

26. Maksym, R. B., Wierzba, W., Baranowski, W. E. P23.06: The feasibility of uterine fibroid treatment with advanced ultrasound-guided HIFU system: preliminary report. Ultrasound in Obstetrics \& Gynecology. 48 (S1), 242-242 (2016).

27. Lee, J. S., Hong, G. Y., Lee, K. H., Song, J. H., Kim, T. E. Safety and efficacy of ultrasound-guided high-intensity focused ultrasound treatment for uterine fibroids and adenomyosis. Ultrasound in Medicine and Biology. 45 (12), 3214-3221 (2019). 
28. Burbank, F. History of uterine artery occlusion and subsequent pregnancy. American Journal of Roentgenology. 192 (6), 1593-1600 (2009).

29. Sack, R. A. Bilateral internal iliac (hypogastric) artery ligation to control obstetric and gynecologic hemorrhage. A ten-year review at the community hospital level. American Journal of Obstetrics and Gynecology. 116 (4), 493-497 (1973).

30. Brown, B. J., Heaston, D. K., Poulson, A. M., Gabert, H. A., Mineau, D. E., Miller, F. J. Uncontrollable postpartum bleeding: A new approach to hemostasis through angiographic arterial embolization. Obstetrics and Gynecology. 54 (3), 361-365 (1979).

31. Heaston, D. K., Mineau, D. E., Brown, B. J., Miller, F. J. Transcatheter arterial embolization for control of persistent massive puerperal hemorrhage after bilateral surgical hypogastric artery ligation. American Journal of Roentgenology. 133 (1), 152-154 (1979).

32. Royal College of Obstetricians and Gynaecologists Clinical recommendations on the use of uterine artery embolization (UAE) in the management of fibroids. Royal College of Radiologists. at <https://www.rcog.org.uk/globalassets/documents/ guidelines/23-12-2013_rcog_rcr_uae.pdf> (2013).

33. Ravina, J. H. et al. Arterial embolization to treat uterine myomata. The Lancet. 346 (8976), 671-672 (1995).

34. Goodwin, S. C., Vedantham, S., McLucas, B., Forno, A. E., Perrella, R. Preliminary experience with uterine artery embolization for uterine fibroids. Journal of Vascular and Interventional Radiology. 8 (4), 517-526 (1997).
35. Kohi, M. P., Spies, J. B. Updates on uterine artery embolization. Seminars in Interventional Radiology. 35 (1), 48-55 (2018).

36. Gupta, J. K., H. M. Cochrane Database of Systematic Reviews Uterine artery embolization for symptomatic uterine fibroids (Review) Uterine artery embolization for symptomatic uterine fibroids (Review). Cochrane Database of Systematic Reviews. (12) (2014).

37. Naredi, N., Bhattacharyya, T. K. Uterine Artery Embolization: A Nonsurgical Cure for Fibroids. South Asian Federation of Obstetrics and Gynecology. 1 (3) (2009).

38. Memtsa, M., Homer, H. Complications associated with uterine artery embolization for fibroids. Obstetrics and Gynecology International. 2012 (290542), 1-5 (2012).

39. Dutton, S., Hirst, A., McPherson, K., Nicholson, T., Maresh, M. A UK multicentre retrospective cohort study comparing hysterectomy and uterine artery embolization for the treatment of symptomatic uterine fibroids (HOPEFUL study): Main results on medium-term safety and efficacy. BJOG: An International Journal of Obstetrics and Gynaecology. 114 (11), 1340-1351 (2007).

40. National Collaborating Centre for Women's and Children's Health (UK) Heavy menstrual bleeding: assessment and management (CG44). Nice guidelines. (January), at <https://www.nice.org.uk/guidance/cg44> (2007).

41. Chen, H. T., Athreya, S. Systematic review of uterine artery embolization practice guidelines: are all the guidelines on the same page? Clinical Radiology. 73 (5), 507.e9-507.e15 (2018). 
42. American College of Obstetricians and Gynecologists ACOG practice bulletin. Alternatives to hysterectomy in the management of leiomyomas. Obstetrics and Gynecology. 112 (2 Pt 1), 387-400 (2008).

43. Carranza-Mamane, B. et al. The Management of Uterine Fibroids in Women With Otherwise Unexplained Infertility. Journal of Obstetrics and Gynaecology Canada. 37 (3), 277-285 (2015).

44. Wozniak, S., Czuczwar, P., Szkodziak, P. R., Pyra, K., Paszkowski, T. EP26.01: Value of "elasto strain ratio" ultrasound elastography in the diagnosis of adenomyosis: preliminary study. Ultrasound in Obstetrics \& Gynecology. 50, 381-381 (2017).

45. Szkodziak, P. R., Wozniak, S., Czuczwar, P., Wrona, W., Trzeciak, K., Paszkowski, T. OP24.04: Value of "elasto strain ratio" ultrasound elastography in the diagnosis of intramural uterine fibroids: preliminary study. Ultrasound in Obstetrics \& Gynecology. 50, 126-126 (2017).

46. Popovic, M., Puchner, S., Berzaczy, D., Lammer, J., Bucek, R. A. Uterine artery embolization for the treatment of adenomyosis: A review. Journal of Vascular and Interventional Radiology. 22 (7), 901-909 (2011).

47. de Bruijn, A. M. et al. Uterine Artery Embolization for the Treatment of Adenomyosis: A Systematic Review and Meta-Analysis. Journal of Vascular and Interventional Radiology. 28 (12) (2017).

48. Bérczi, V. et al. Safety and Effectiveness of UFE in Fibroids Larger than $10 \mathrm{~cm}$. CardioVascular and Interventional Radiology. 38 (5), 1152-1156 (2015).

49. Stępniak, A. Uterine artery embolization in the treatment of symptomatic fibroids - State of the art 2018. Przeglad Menopauzalny. 17 (4), 141-143 (2018).
50. Czuczwar, P. et al. The influence of uterine artery embolization on ovarian reserve, fertility, and pregnancy outcomes - A review of literature. Przeglad Menopauzalny. 15 (4), 205-209 (2016).

51. Firouznia, K., Ghanaati, H., Sanaati, M., Jalali, A. H., Shakiba, M. Pregnancy after uterine artery embolization for symptomatic fibroids: A series of 15 pregnancies. American Journal of Roentgenology. 192 (6), 1588-1592 (2009).

52. Bonduki, C. E. et al. Pregnancy after uterine arterial embolization. Clinics. 66 (5), 807-810 (2011).

53. Yeaton-Massey, A., Loring, M., Chetty, S., Druzin, M. Uterine rupture after uterine artery embolization for symptomatic leiomyomas. Obstetrics and Gynecology. 123 (2 PART 2), 418-420 (2014).

54. Takahashi, H., Hayashi, S., Matsuoka, K., Kitagawa, M. Placenta accreta following uterine artery embolization. Taiwanese Journal of Obstetrics and Gynecology. 49 (2), 197-198 (2010).

55. Takeda, J. et al. Spontaneous uterine rupture at 32 weeks of gestation after previous uterine artery embolization. Journal of Obstetrics and Gynaecology Research. 40 (1), 243-246 (2014).

56. Practice Committee of American Society for Reproductive Medicine in collaboration with Society of Reproductive Surgeons Myomas and reproductive function. Fertility and Sterility. 90 (5 Suppl.), 125-130 (2008).

57. National Institute for Health and Clinical Excellence (NICE) Uterine artery embolization for fibroids (Interventional procedures guidance [IPG367]). Nice Guidelines. at <http://publications.nice.org.uk/uterineartery-embolization-for-fibroids-ipg367> (2010). 
58. Leibsohn, S., d'Ablaing, G., Mishell, D. R., Schlaerth, J. B. Leiomyosarcoma in a series of hysterectomies performed for presumed uterine leiomyomas. American Journal of Obstetrics and Gynecology. 162 (4), 968-976 (1990).

59. Parker, W. H., Fu, Y. S., Berek, J. S. Uterine sarcoma in patients operated on for presumed leiomyoma and rapidly growing leiomyoma. Obstetrics and Gynecology. 83 (3), 414-418, at <https://pubmed.ncbi.nlm.nih.gov/8127535/ $>$ (1994).

60. Ricci, S., Stone, R. L., Fader, A. N. Uterine leiomyosarcoma: Epidemiology, contemporary treatment strategies and the impact of uterine morcellation. Gynecologic Oncology. 145 (1), 208-216 (2017).

61. Felix, A. S. et al. The etiology of uterine sarcomas: A pooled analysis of the epidemiology of endometrial cancer consortium. British Journal of Cancer. 108 (3), 727-734 (2013).

62. Roberts, M. E., Aynardi, J. T., Chu, C. S. Uterine leiomyosarcoma: A review of the literature and update on management options. Gynecologic Oncology. 151 (3), 562-572 (2018).

63. Schieda, N. et al. Gadolinium-Based Contrast Agents in Kidney Disease: A Comprehensive Review and Clinical Practice Guideline Issued by the Canadian Association of Radiologists. Canadian Journal of Kidney Health and Disease. 5, 1-17 (2018).

64. Pyra, K. et al. Uterine artery embolization for the treatment of symptomatic uterine fibroids. (Embolizacja tętnic macicznych w leczeniu objawowych mięśniaków macicy.). Postępy Nauk Medycznych. 28 (2), 88-94, at <http://www.pnmedycznych.pl/wp-content/ uploads/2015/03/pnm_2015_088-094.pdf> (2015).
65. Czuczwar, P., Stepniak, A., Milart, P., Paszkowski, T., Wozniak, S. Comparison of the influence of three fibroid treatment options: Supracervical hysterectomy, ulipristal acetate and uterine artery embolization on ovarian reserve - An observational study. Journal of Ovarian Research. 11 (1) (2018).

66. Nocum, D. J., Robinson, J., Liang, E., Thompson, N., Reed, W. The factors contributing to the total radiation exposure of patients during uterine artery embolization. Journal of Medical Radiation Sciences. 66 (3), 200-211 (2019).

67. Nikolic, B., Spies, J. B., Lundsten, M. J., Abbara, S. Patient radiation dose associated with uterine artery embolization. Radiology. 214 (1), 121-125 (2000).

68. Colgan, T. J. et al. Pathologic features of uteri and leiomyomas following uterine artery embolization for leiomyomas. The American Journal of Surgical Pathology. 27 (2), 167-77, at <http:// www.ncbi.nlm.nih.gov/pubmed/12548162> (2003).

69. Weichert, W. et al. Uterine arterial embolization with trisacryl gelatin microspheres: a histopathologic evaluation. The American Journal of Surgical Pathology. 29 (7), 95561, at <http://www.ncbi.nlm.nih.gov/pubmed/15958862> (2005).

70. Walker, W. J., Pelage, J. P. Uterine artery embolization for symptomatic fibroids: clinical results in 400 women with imaging follow up. BJOG: An International Journal of Obstetrics and Gynaecology. 109 (11), 126272, at <http://www.ncbi.nlm.nih.gov/pubmed/12452465> (2002).

71. Ravina, J.H., Aymard, A., Ciraru-Vigneron, N., Clerissi, J., Merland, J. J. Uterine fibroids embolization: results about 454 cases. Gynécologie, Obstétrique \& Fertilité. 
31 (7-8), 597-605, at <http://www.ncbi.nlm.nih.gov/ pubmed/14563603> (2003).

72. Czuczwar, P. et al. Predicting the results of uterine artery embolization: Correlation between initial intramural fibroid volume and percentage volume decrease. Przeglad Menopauzalny. 13 (4), 247-252 (2014).

73. Lohle, P. N. M. et al. Long-term Outcome of Uterine Artery Embolization for Symptomatic Uterine Leiomyomas. Journal of Vascular and Interventional Radiology. 19 (3), 319-326 (2008).

74. Walker, W. J., Barton-Smith, P. Long-term follow up of uterine artery embolization - An effective alternative in the treatment of fibroids. BJOG: An International Journal of Obstetrics and Gynaecology. 113 (4), 464-468 (2006).

75. Czuczwar, P. et al. Influence of ulipristal acetate therapy compared with uterine artery embolization on fibroid volume and vascularity indices assessed by threedimensional ultrasound: Prospective observational study. Ultrasound in Obstetrics and Gynecology. 45 (6), 744750 (2015).

76. Van Der Kooij, S. M. et al. Epidural analgesia versus patient-controlled analgesia for pain relief in uterine artery embolization for uterine fibroids: A decision analysis. CardioVascular and Interventional Radiology. 36 (6), 1514-1520 (2013).

77. Pisco, J. M. et al. Pelvic Pain after Uterine Artery Embolization: A Prospective Randomized Study of Polyvinyl Alcohol Particles Mixed with Ketoprofen versus Bland Polyvinyl Alcohol Particles. Journal of Vascular and Interventional Radiology. 19 (11), 1537-1542 (2008).

78. Pisco, J. M. et al. Uterine Artery Embolization under Electroacupuncture for Uterine Leiomyomas. Journal of
Vascular and Interventional Radiology. 20 (7), 863-870 (2009).

79. Pelage, J.P. et al. Uterine embolization. Journal de Radiologie. 81 (12 Suppl), 1873-1874, at <http:// www.ncbi.nlm.nih.gov/pubmed/11173757> (2000).

80. Pelage, J.P. et al. Uterine Fibroid Vascularization and Clinical Relevance to Uterine Fibroid Embolization. RadioGraphics. 25 (suppl_1), S99-S117 (2005).

81. Siskin, G. P. et al. Outpatient uterine artery embolization for symptomatic uterine fibroids: Experience in 49 patients. Journal of Vascular and Interventional Radiology. 11 (3), 305-311 (2000).

82. Salehi, M., Jalilian, N., Salehi, A., Ayazi, M. Clinical Efficacy and Complications of Uterine Artery Embolization in Symptomatic Uterine Fibroids. Global Journal of Health Science. 8 (7) (2015).

83. Worthington-Kirsch, R. et al. The Fibroid Registry for outcomes data (FIBROID) for uterine embolization: Shortterm outcomes. Obstetrics and Gynecology. 106 (1), 5259 (2005).

84. Paszkowski, T. et al. Uterine artery embolization - clinical problems. Polish Gynaecology. 84 (12) (2013).

85. Spies, J. B. et al. Fibroid Registry Investigators. The FIBROID Registry: symptom and quality-of-life status 1 year after therapy. Obstetrics \& Gynecology. 106 (6), 1309-1318 (2005).

86. Chrisman, H.B. et al. The impact of uterine fibroid embolization on resumption of menses and ovarian function. Journal of Vascular and Interventional Radiology. 11 (6), 699-703 (2000).

87. Maciel, C., Tang, Y. Z., Sahdev, A., Madureira, A. M., Vilares-Morgado, P. Preprocedural MRI and 
MRA in planning fibroid embolization. Diagnostic and Interventional Radiology. 23 (2), 163-171 (2017).

88. Laughlin-Tommaso, S. K. et al. Clinical limitations of the International Federation of Gynecology and Obstetrics (FIGO) classification of uterine fibroids. International Journal of Gynecology and Obstetrics. 139 (2), 143-148 (2017).

89. Vilos, G. A. Uterine fibroids: relationships to reproduction. Minerva Ginecologica. 55 (5), 417-23, at <http:// www.ncbi.nlm.nih.gov/pubmed/14581884> (2003).

90. McLucas, B. Pregnancy following uterine artery embolization: An update. Minimally Invasive Therapy and Allied Technologies. 22 (1), 39-44 (2013).

91. Karlsen, K., Hrobjartsson, A., Korsholm, M., Mogensen, O., Humaidan, P., Ravn, P. Fertility after uterine artery embolization of fibroids: a systematic review. Archives of Gynecology and Obstetrics. 297 (1), 13-25 (2018).

92. Margau, R. et al. Outcomes after Uterine Artery Embolization for Pedunculated Subserosal Leiomyomas. Journal of Vascular and Interventional Radiology. 19 (5), 657-661 (2008).

93. Spies, J. B. et al. The FIBROID registry: Symptom and quality-of-life status 1 year after therapy. Obstetrics and Gynecology. 106 (6), 1309-1318 (2005).

94. Avilla-Hernandez, Adrian; Singh, P. Epidural Anesthesia - StatPearls - NCBI Bookshelf. Statpearls. 1, at <https://www.ncbi.nlm.nih.gov/books/NBK542219/? report=classic > (2019).

95. Higgs, Z. C. J., Macafee, D. A. L., Braithwaite, B. D., Maxwell-Armstrong, C. A. The Seldinger technique: 50 Years on. Lancet. 366 (9494), 1407-1409 (2005).
96. Seldinger, S. I. Catheter replacement of the needle in percutaneous arteriography: A new technique. Acta Radiologica. 39 (5), 368-376 (1953). 